Original Paper http://ajol.info/index.php/ijbcs http://indexmedicus.afro.who.int

\title{
Taxonomie du genre Ficus au Sénégal : apport des caractères morphologiques
}

\author{
Doudou DIOP ${ }^{*}$, Mame Samba MBAYE ${ }^{2}$, Seyni $\mathrm{SANE}^{2}$, Kandioura NOBA ${ }^{2}$ \\ et Amadou Tidiane BA ${ }^{2}$ \\ ${ }^{1}$ Laboratoire de Botanique, Institut Fondamental d'Afrique Noire/CAD, Dakar, Sénégal. \\ ${ }^{2}$ Laboratoire de Botanique et Biodiversité, Département de Biologie végétale, UCAD, Dakar, Sénégal. \\ *Auteur correspondant ; E-mail: doudou.diop@ucad.edu.sn ; Tél : (+221)775716672.
}

\section{RÉSUMÉ}

Le genre Ficus L. (Moraceae) constitue le groupe ligneux le plus diversifié de la Flore du Sénégal; il compte plus de 30 espèces. Les études antérieures ont amélioré la caractérisation de nombreuses espèces mais elles n'ont pas permis de résoudre toutes les difficultés taxonomiques rencontrées chez le genre Ficus. Cette étude porte sur les caractères morphologiques des espèces du genre, des feuilles et des figues en particulier, afin d'identifier les caractères pertinents qui permettent d'améliorer leur détermination, pour in fine proposer une clé d'identification. Elle porte sur 24 taxa de la Flore du Sénégal. Des méthodes d'études qualitatives et quantitatives ont été appliquées aux ports, aux modes de développement, aux racines adventives, aux feuilles et figues. Les résultats de cette étude ont permis de préciser l'importance taxonomique des caractères tels que : la présence ou l'absence des racines aériennes, des éperons, des calyptres et la disposition des figues et de ses organes reproducteurs. En outre, cette étude a permis la discrimination de deux variétés de $F$. glumosa et des deux espèces $F$. iteophylla et $F$. thonningii souvent considérées comme des synonymes. Enfin, une nouvelle clé, commode et précise pour détermination des 24 taxa étudiés, a été proposée.

(c) 2018 International Formulae Group. All rights reserved.

Mots clés : Moraceae, Ficus, morphologie, figue, taxonomie.

\section{Taxonomy of the genus Ficus in Senegal: contribution of morphological characters}

\begin{abstract}
The genus Ficus is the most diversified ligneous group of the Senegalese flora, with more than 30 species. Previous studies have improved the characterization of many species but could not resolve all the taxonomical issues raised by the genus Ficus. This study relates to the morphological characteristics of the genus species of the sheets and figs in particular and aims at identifying the discriminants allowing to improve their determination so as to provide identification keys. It relates to 24 taxa of the Flora in Senegal. Methods of qualitative and quantitative studies have been applied to the port, to modes of development, to adventitious roots, to sheets and figs. The results of this
\end{abstract}


study allowed to specify the taxonomical importance of characters such as: the presence or absence of aerial roots, spurs and calyptrates, and the set up of figs on the plant and of its reproductive organs. Moreover, it allowed to specify the discriminating characteristics of the varieties of $F$. glumosa, and to differentiate the species $F$. iteophylla and $F$. thonningii, commonly considered as synonymous. Lastly, this study enables to offer a new key which is more precise and convenient to determine the 24 studied taxa.

(c) 2018 International Formulae Group. All rights reserved.

Keywords: Moraceae, Ficus, fig, morphology, taxonomy.

\section{INTRODUCTION}

Le genre Ficus L., de distribution pantropicale, est l'un des plus importants de la flore tropicale par le nombre d'espèces. Le genre Ficus compte 755 à 800 espèces dont 500 pour la région Asie-Australienne. En Afrique, 105 espèces sont présentes dans la zone tropicale et 70 parmi elles sont signalées en Afrique de l'Ouest (Loutfy et al., 2005 ; Diop, 2013 ; Adeoluwa et al., 2014).

La taxonomie des Ficus africains a été révisée par Berg et Wiebes (1992) et les grandes lignes de cette classification, notamment la subdivision en sous-genres et en sections, ont été majoritairement acceptées. Toutefois, il est important de souligner que leurs clés d'identification n'ont pas permis de résoudre les nombreuses confusions taxonomiques qui sont souvent à l'origine de plusieurs synonymies notées chez ce genre. Une révision de ces travaux partant de ces identifications en herbier devrait permettre de corriger les quelques erreurs et d'identifier des caractères nouveaux pour améliorer la reconnaissance des espèces du genre Ficus.

La récente tentative de mise à jour de cette révision a été celle de Burrows et Burrows (2003) mais elle portait sur une petite zone se limitant aux régions Sud et Centrales de l'Afrique.

Au Sénégal comme dans la plupart des régions tropicales et subtropicales, les espèces du genre Ficus jouent un rôle central dans le fonctionnement des écosystèmes en favorisant la survie de beaucoup d'animaux consommateurs de figues et disperseurs de graines (Beaune et al., 2013 ; Cottee-Jone et al., 2016). Toutefois, l'utilisation de ces essences forestières est limitée par l'insuffisance des informations obtenues sur ces espèces locales et notamment celles qui ont trait à leur taxonomie où des confusions sont notées aussi bien dans le complexe thonningii que chez d'autres espèces. D'où la nécessité d'aborder cette étude sur la taxonomie des espèces du genre Ficus rencontrées au Sénégal. Elle a pour objectif de réviser les caractères morphologiques de chacune des espèces étudiées afin mieux préciser son identité. Pour ce faire, cette étude propose de :

- revisiter les caractères taxonomiques de l'appareil végétatif et ceux de l'appareil reproducteur ;

- rechercher des caractères discriminants additionnels au plan morphologique susceptibles d'améliorer la détermination des espèces et de proposer une nouvelle clé d'identification.

\section{MATÉRIEL ET MÉTHODES Matériel}

Le matériel végétal utilisé provient : - des récoltes effectuées au Sénégal dans les régions de Dakar, Fatick, Kédougou, Kolda, Saint-Louis, Tambacounda, Thiès et de Ziguinchor ;

- des herbiers du laboratoire de Botanique de I'IFAN Cheikh Anta Diop de l'UCAD, Dakar et du Département de Biologie Végétale de la Faculté des Sciences et Techniques de l'UCAD, Dakar.

Les espèces étudiées ont été identifiées en utilisant les flores de Berhaut (1967), Berg et al. (1985) et de Arbonnier 
(2009). Pour chacune des espèces étudiées, des échantillons d'herbiers fertiles ont été récoltés puis séchés à l'ombre, à l'abri de l'humidité et de la chaleur, en prenant soin de trier chacun de leurs organes (feuilles, fruits, écorce et racines) qui sont ensuite conservés soigneusement jusqu'à leur étude. Une autre partie de cette récolte a été utilisée pour le montage des échantillons d'herbiers.

Du matériel frais a été récolté et conservé dans du F.A.A. (Formol 5ml, Acide acétique $5 \mathrm{ml}$ et Ethanol $70^{\circ} 90 \mathrm{ml}$ ). Il a été utilisé pour les études morphométriques et morphologiques.

Le matériel de laboratoire utilisé est composé de :

- loupe binoculaire (loupe Wild M 32, marque Leica) ;

- microscope optique (marque Euromex) ; pied à coulisse.

\section{Méthodes d'études des caractères morphologiques}

L'étude morphologique a été menée par l'observation et la description des différentes parties aériennes des plantes adultes.

\section{Morphologie des organes végétatifs}

L'étude a porté sur le port, le mode de développement, les racines adventives et les feuilles. Elle a été réalisée à partir d'observations faites sur le terrain dans différentes localités d'une ou des différentes régions administratives prospectées, et sur des spécimens collectés et destinés à être conservés dans des herbiers.

Le type de port, le mode de développement et la présence ou non des racines adventives, ont été notés à chaque fois qu'une espèce est rencontrée sur le terrain.

L'étude comparée de la morphologie des feuilles des différentes espèces étudiées a porté sur les caractères qualitatifs du limbe (forme, sommet, base, marges, pubescence, rugosité, flexibilité) et quantitatifs (longueur et largeur) supposés pertinents pour la caractérisation des espèces étudiées.

\section{Morphologie des organes reproducteurs Méthodes d'études qualitatives des figues}

Une description morphologique des figues a été faite grâce à des observations à l'œil nu et à la loupe binoculaire. Une observation sur des coupes perpendiculaires à l'axe ostiole - pédoncule des figues a permis de déterminer la position des fleurs mâles à l'intérieur des figues.

\section{Méthodes d'études quantitatives des figues}

Une étude biométrique a été réalisée sur les figues, en état de fruits composés, pour comparer les caractères quantitatifs des figues des différentes espèces étudiées et en déduire l'importance systématique.

L'étude a porté sur les caractères quantitatifs suivants : la distance ostiole-base des figues, appelée longueur (L), le diamètre (D) de la figue et la longueur du pédoncule (Lp). Pour cela, 53 figues de chaque espèce sont collectées au hasard et les différents variables $(\mathrm{D}, \mathrm{L}, \mathrm{Lp})$ sont mesurés et analysés.

Une analyse de variance (ANOVA) a été réalisée grâce au logiciel $\mathrm{R}$ (Dagnelie, 2011-2013; R Core Team, 2018) pour comparer les caractéristiques quantitatives des différentes espèces de figue. Un test de Newman Keuls (Abdi \& Williams, 2010) de structuration des moyennes est fait à la suite de l'ANOVA pour comparer les espèces deux à deux quand le test d'ANOVA est significatif. Le seuil de significativité est fixé à $5 \%(\mathrm{p}<0.05)$. La nomenclature utilisée dans ce travail est basée sur les travaux de Lebrun et Stork (1992).

\section{RÉSULTATS}

Ces études portent en premier lieu sur la morphologie générale des différentes espèces étudiées puis sur les caractères de la feuille et de la figue.

\section{Morphologie générale}

Les observations sur le type de port, le mode de développement et la présence ou non de racines aériennes des différentes espèces étudiées du genre Ficus sont mentionnées dans le Tableau 
L'analyse des résultats de ce Tableau 1 montre que les espèces présentent deux modes de développement :

- un développement hémi-épiphyte chez les espèces suivantes : F. conraui, F. elasticoïdes, $F$. iteophylla, $F$. lutea, $F$. natalensis, $F$. ottoniifolia, $F$. ovata, $F$. polita, $F$. thonningii, F. trichopoda, F. scott-eliotii et $F$. umbellata; - un développement sans phase épiphytique chez les autres que sont: F. abutilifolia, $F$. capreaefolia, $F$. cordata, $F$. dicranostyla, $F$. exasperata, $F$. glumosa var. glaberrima, $F$. glumosa var. glumosa, $F$. platyphylla, $F$. sur, $F$. sycomorus et $F$. vallis-choudae.

Chez les espèces à développement hémi-épiphytique, seules $F$. conraui, $F$. natalensis et $F$. ottoniifolia présentent un port arbustif avec en général un tronc court ramifié à ras du sol donnant ainsi à ces espèces un aspect buissonnant.

Concernant les espèces à développement non-épiphyte, le port buissonnant permet de singulariser les espèces $F$. capreaefolia et $F$. cordata tandis que le port arbustif observé chez $F$. abutilifolia, $F$. exasperata et $F$. sur, permet de distinguer ces 3 espèces des autres à port arborescent.

\section{Morphologie de la feuille}

L'étude morphologique de la feuille a porté sur les caractères taxonomiques les plus importants chez les espèces du genre Ficus. Deux types de caractères ont été étudiés :

- les caractères qualitatifs :

- du limbe tels que la forme, la marge, le sommet, la base, la souplesse, la pubescence et la sensation au toucher (lisse ou scabre) ;

- du pétiole tels que la pubescence et la sensation au toucher;

- et les caractères quantitatifs du limbe (longueur et largeur).

Les résultats obtenus sont répertoriés dans le Tableau 2.

Les résultats obtenus montrent qu'en plus des caractères communs (feuilles simples, pétiolées, alternes, exceptionnellement subopposées chez $F$. capreaefolia (Figure 2)), il existe d'autres caractères différentiels qui peuvent faciliter l'identification des espèces étudiées. Certains de ces caractères sont propres au limbe alors que d'autres sont liés au pétiole. Parmi les caractères propres au limbe, la forme permet de distinguer les espèces à feuilles ovales à elliptiques des espèces à feuilles non ovales.

Chez les espèces à feuilles non ovales, nous pouvons distinguer les espèces à feuilles suborbiculaires des espèces à feuilles obovales.

- Chez les espèces à feuilles suborbiculaires, les résultats montrent 2 espèces $(F$. abutlifolia et $F$. umbellata: Figure 1 et Figure 24) présentant un limbe cordé, glabre, lisse et à marge rectiligne. Toutefois $F$. umbellata présente un limbe légèrement acuminé de plus grande taille $(17,3-23,0 \mathrm{~cm})$ contrairement à F. abutilifolia qui a un limbe non acuminé et de plus petite taille $(6,7-9,3 \mathrm{~cm})$.

- Chez les espèces à feuilles obovales, la forme obtriangulaire, le sommet aplati et la petite taille du limbe $(4,9-7,7 \mathrm{~cm})$ de $F$. natalensis (Figure 13) permettent de la différencier d'emblée de l'espèce $F$. conraui (Figure 3) qui a un limbe obovale à sommet acuminé et de longueur nettement plus importante $(17,6-25 \mathrm{~cm})$.

Hormis ces 4 espèces citées cidessus, les autres espèces étudiées ont un limbe ovale ou elliptique.

Chez ces espèces à feuilles ovales à elliptiques, les caractères qualitatifs du limbe permettent de distinguer :

- quatre espèces présentant un limbe scabre sur au moins une des deux faces; il s'agit de :

- d'une part de $F$. dicranostylla et de $F$. sycomorus (Figure 5 et Figure 20) dont la face supérieure de la feuille est scabre contrairement à celle inferieure qui est lisse ; - d'autre part de $F$. capreaefolia et $F$. exasperata (Figure 2 et Figure 7) qui ont des feuilles entièrement scabres; le limbe elliptique de $F$. capraefolia et sa base aigue permettent de discriminer cette espèce de $F$. exasperata qui présente un limbe plus large de forme ovale à base légèrement cordée ;

- deux espèces dont la marge du limbe est irrégulière; il s'agit de $F$. sur et $F$. vallis- 
choudae (Figure 24) qui présentent respectivement une marge dentée et une marge sinueuse au 2/3 supérieur ;

- les autres espèces à feuilles ovales à elliptiques présentent un limbe non scabre avec une marge rectiligne; parmi ces dernières, le caractère quantitatif lié à la taille du limbe permet d'observer 2 groupes :

- un groupe d'espèces à feuilles de taille moyenne représenté $F$. elasticoüdes, $F$. lutea, $F$. ottoniifolia, F. ovata, F. platyphylla, $F$. polita et $F$. trichopoda ;

- un groupe d'espèces à feuilles de petite taille représenté par $F$. cordata, $F$. glumosa var. glaberrima, $F$. glumosa var. glumosa, $F$. ingens, $F$. scott-eliotii, $F$. thonningii et $F$. iteophylla.

Dans le groupe d'espèces à feuilles de taille moyenne, la forme de la base du limbe permet de distinguer :

- des feuilles cordées chez quatre espèces : $F$. platyphylla, $F$. ovata, $F$. polita et $F$. trichopoda (Figure 15; Figure 16; Figure 17 et Figure 22); le limbe longuement acuminé de $F$. polita permet de la singulariser des trois autres espèces dont le sommet du limbe est obtus ; chez les 3 autres espèces, la pubescence du limbe de $F$. platyphylla permet de distinguer celle-ci de $F$. ovata et de $F$. trichopoda; cette dernière présente un limbe plus cordé mais de taille inférieure à celle de F. ovata;

- des feuilles non cordées chez trois espèces ; parmi celles-ci, deux espèces $F$. elasticoüdes et $F$. ottoniifolia (Figure 6 et Figure. 14) présentent un limbe elliptique qui est: coriace, large à sommet obtus chez $F$. elasticoides tandis qu'il est souple et acuminé chez $F$. ottoniifolia; la troisième espèce, $F$. lutea, a un limbe ovale à elliptique avec une base arrondie par opposition à la base aiguë de $F$. elasticoüdes et $F$. ottoniifolia.

Dans le groupe d'espèces à feuilles de petite taille, la forme de la base du limbe permet également de distinguer :

- des feuilles cordées chez $F$. cordata, $F$. glumosa var. glaberrima, $F$. glumosa var. glumosa, F. ingens (Figure 4 ; Figure 8 ;
Figure 9 et Figure 10); F. cordata se singularise par son limbe acuminé et de plus petite dimension par rapport aux autres tandis que $F$. ingens est la seule espèce qui a un limbe forme pyramidale; les caractères étudiés de la feuille ne permettent pas de séparer les 2 variétés de glumosa;

- des feuilles non cordées chez F. scott-eliotii, $F$. iteophylla et $F$. thonningii (Figure 18 ; Figure 11 et Figure 21) ; 1'espèce $F$. scotteliotii diffère des deux autres par la base arrondie du limbe et sa rigidité ; les caractères étudiés ne permettent pas de discriminer $F$. iteophylla de $F$. thonningii.

\section{Morphologie des organes reproducteurs Caractères qualitatifs des figues}

L'étude morphologique des figues porte sur les caractères qualitatifs suivants : localisation sur la plante, disposition, forme, présence ou non de pédoncules sur les figues, pubescence, sensation au toucher, forme de l'ostiole, visibilité des bractéoles (bractées du réceptacle), présence ou non d'éperons sur les branches ou rameaux, présence ou non de calyptres à l'aisselle des feuilles et à la position des fleurs mâles dans le réceptacle urcéolé.

Les résultats obtenus sont mentionnés dans le Tableau 3.

L'analyse des résultats montre que la présence ou l'absence du pédoncule permet de diviser les espèces étudiées en deux groupes : - un groupe d'espèces à figues nettement sessiles à subsessiles formé par : F. conraui, $F$. cordata, $F$. ingens, $F$. lutea, $F$. ovata, $F$. thonningii, $F$. umbellata et $F$. vallis-choudae; - un groupe d'espèces à figues pédonculées formé par: $F$. abutilifolia, $F$. capreaefolia, F. dicranostyla, F. elasticoides, $F$. exasperata, $F$. glumosa var. glaberrima, $F$. glumosa var. glumosa, $F$. iteophylla, $F$. natalensis, $F$. ottoniifolia, $F$. platyphylla, $F$. polita, F. scott-ellioti, F. sur, F. sycomorus et F. trichopoda.

Concernant les espèces du premier groupe, nous pouvons observer :

- des espèces à figues nettement sessiles. Il s'agit de $F$. conraui, $F$. lutea et $F$. thonningii ; 
parmi ces espèces, seule $F$. lutea a des figues pubescentes; $F$. conraui et $F$. thonningii ont en commun les caractères qualitatifs étudiés ;

- et des espèces avec figues courtement pédonculées ou subsessiles. Il s'agit de $F$. cordata, $F$. ingens, $F$. ovata, $F$. umbellata et $F$. vallis-choudae. Dans ce groupe d'espèces nous pouvons distinguer :

- $F$. cordata, $F$. ingens et $F$. vallis-choudae par les caractères liés à la forme circulaire de l'ostiole des figues et par la position des fleurs mâles ; $F$. ingens peut se singulariser par la pubescence des figues avec un éperon à la base du pédoncule et $F$. vallis-choudae par le caractère solitaire de ses figues à l'aisselle des feuilles.

- F. ovata et $F$. umbellata par la forme en fente de l'ostiole des figues et la dissémination des fleurs mâles sur toute la surface interne des figues; toutefois, $F$. ovata et $F$. umbellata se distinguent l'une de l'autre respectivement par la présence de calyptres bien développés à l'aisselle des feuilles et par la présence d'éperons sur les rameaux.

Concernant les espèces à figues pédonculées, les caractères étudiés permettent de distinguer :

- La cauliflorie chez F. sur dont des figues sphériques sont sur des ramilles aphylles du tronc et des branches ;

- la présence d'éperons à la base des pédoncules chez $F$. ottoniafolia et $F$. polita ; toutefois, on peut reconnaître $F$. ottoniifolia par la forme ovoïde de ses figues tandis que $F$. polita, cauliflore stricte, présente des figues sphériques disposées sur de grosses branches et non à l'aisselle des feuilles ;

- la rugosité de leurs figues chez $F$. capreaefolia et $F$. exasperata ;

- la pubescence des figues chez $F$. glumosa var. glumosa, $F$. iteophylla et $F$. sycomorus ; la forme circulaire de l'ostiole permet de singulariser $F$. sycomorus ;

- une disposition en grappe des figues à l'extrémité des rameaux chez $F$. abutilifolia, $F$. glumosa var. glaberrima, F. glumosa var. glumosa et $F$. platyphylla ;

- une disposition des figues par paire à l'aisselle des pétioles chez $F$. dicranostyla, $F$. elasticoides, $F$. natalensis, $F$. scott-ellioti et $F$. trichopoda.

\section{Caractères quantitatifs des figues}

Les moyennes et les intervalles de confiance des 53 mesures effectuées pour chaque variable sont mentionnés dans le Tableau 4.

L'analyse du Tableau 4 montre que les dimensions des figues sont très variables. Le traitement statistique (ANOVA; test de Newman et Keuls) des mesures des différents variables, avec un seuil de significativité $(\mathrm{p})<$ $5 \%$, permet de distinguer 3 groupes d'espèces selon la longueur moyenne du pédoncule (Lp.):

- un groupe d'espèces à figues sessiles constitué de $F$. conraui, $F$. lutea et $F$. thonningii. Ces espèces se différencient par le diamètre moyen des figues : 19,13 $\pm 1,53 \mathrm{~mm}$ pour $F$. conraui ; $15,24 \pm 1,53 \mathrm{~mm}$ pour $F$. lutea et $9,54 \pm 1,51 \mathrm{~mm}$ pour $F$. thonningii ;

- un groupe d'espèces à figues subsessiles ou courtement pédonculées $(0 \leq$ Lp. $\leq 4,96 \pm$ $0,83 \mathrm{~mm}$ ) représenté par 2 espèces, $F$. cordata et $F$. ingens qui présentent de petites figues avec un diamètre inférieur à $11,09 \pm 1,51 \mathrm{~mm}$ et 2 autres espèces, $F$. ovata et $F$. vallischoudae dont le diamètre des figues est plus important avec respectivement $27,85 \pm 1,52$ mm et $46,23 \pm 1,43 \mathrm{~mm}$ de diamètre ;

- un groupe d'espèces à figues nettement pédonculées (Lp. $\geq 6,5 \pm 0,84 \mathrm{~mm}$ ) que sont : $F$. abutilifolia, $F$. capreaefolia, $F$. dicranostyla, $F$. elasticoïdes, $F$. exasperata, $F$. glumosa var. glaberrima, $F$. glumosa var. glumosa, $F$. iteophylla, $F$. natalensis, $F$. ottoniifolia, F. platyphylla, F. polita, F. scotteliotii, $F$. sur, F. sycomorus, $F$. trichopoda et $F$. umbellata. Dans ce groupe, les caractères liés aux dimensions permettent de distinguer des espèces dont les figues ont:

- de petits diamètres $(6,42 \pm 1,53 \mathrm{~mm} \leq \mathrm{D} \geq$ $12,88 \pm 1,51 \mathrm{~mm}$ ) telles que $F$. abutilifolia, $F$. capreaefolia, $F$. dicranostyla, $F$. exasperata, $F$. glumosa var. glaberrima, F. glumosa var. glumosa, $F$. iteophylla, $F$. natalensis, $F$. ottoniifolia, $F$. platyphylla et $F$. trichopoda. 
Parmi ces espèces, certaines présentent des figues pédonculées de longueur :

- supérieure à 12,05 $\pm 0,83 \mathrm{~mm}$; il s'agit de $F$. abutilifolia, $F$. capreaefolia, $F$. dicranostyla, $F$. ottoniifolia et $F$. platyphylla;

- inferieure à 9,93 $\pm 0,82 \mathrm{~mm}$; il s'agit de $F$. glumosa var. glaberrima, $F$. glumosa var. glumosa, F. iteophylla, $F$. natalensis et $F$. trichopoda.

- des diamètres moyens $(17,5 \pm 1,52 \mathrm{~mm} \leq \mathrm{D}$ $\geq 30,94 \pm 1,51 \mathrm{~mm})$; il s'agit de $F$. elasticoïdes, F. polita, F. sur, F. scott-eliotii, $F$. sycomorus et $F$. umbellata. Nous pouvons distinguer dans ce groupe, des espèces dont la longueur :

- du pédoncule est supérieure à 14,5 \pm $0,82 \mathrm{~mm}$ : F. elasticoïdes, $F$. polita, $F$. sur et F. sycomorus ;

- du pédoncule est inférieure à $9,90 \pm 0,52$ mm: $F$. scott-eliotii et $F$. umbellata.

\section{DISCUSSION}

\section{Morphologie de l'appareil végétatif}

Les résultats ont montré l'absence des racines adventives chez les quatre espèces non épiphytes : $F$. abutilifolia, $F$. dicranostyla, $F$. sur et $F$. sycomorus. L'absence de racines adventives est un caractère rare chez les figuiers (Diop, 2013). Il constitue, de ce fait, un caractère taxonomique important.

Le type de port buissonnant de $F$. capreafolia et $F$. cordata permet de distinguer ces deux espèces aux autres espèces dont le port est arbustif ou arborescent.

Le mode de développement des espèces étudiées a permis de distinguer 2 groupes de taxa: les espèces hémi-épiphytes et les espèces non-épiphytes. Les caractères liés au mode de développement ont été souvent utilisés chez le genre Ficus et constituent de bons critères distinctifs de taxon (Zhekun and Gilbert, 2003, Kumar et al., 2011 ; Diop, 2013).

La combinaison des caractères morphologiques liés au port, au mode développement et à la présence ou non de racines adventives chez les espèces du genre Ficus a été souvent utilisée par certains auteurs dans les clés de déterminations (Kumar et al., 2011 ; Diop, 2013 ; Dhungana et al., 2015).

$\mathrm{Au}$ total, les caractères étudiés portant sur le port et le mode de développement ont permis, d'une part, de suspecter l'identité $F$. capreafolia et $F$. cordata, et d'autre part, de distinguer les groupes d'espèces hémi-épiphytes et non épiphytes au sein desquelles un sous-groupe se singularise par l'absence de racines adventives. Ces caractères étudiés peuvent donc être considérés comme des critères importants dans la détermination des espèces étudiées. Cependant, ils restent insuffisants pour une détermination précise des espèces du genre Ficus. Ainsi, l'étude des caractères de la feuille a été envisagée afin de trouver d'autres caractères distinctifs entre les différentes espèces étudiées.

Les résultats obtenus de l'étude morphologique de la feuille montrent la pertinence du choix des caractères qualitatifs (formes du limbe, de sa marge, de son sommet, base, flexibilité ou rigidité, pilosité, sensation au toucher du limbe et du pétiole) et quantitatifs du limbe (longueur, largeur) pour identifier les espèces du genre Ficus L.

A l'échelle interspécifique :

- La forme du limbe, son sommet et sa base ont permis de distinguer sans difficulté les 3 groupes d'espèces suivants : les espèces avec un limbe ovale-elliptique, les espèces à feuilles suborbiculaires et les espèces à feuilles obovales. Les formes particulières du limbe des espèces $F$. conraui, $F$. ingens et $F$. natalensis (Figure 3 ; Figure 10 et Figure 13) permettent de suspecter d'emblée leurs identités. La forme du limbe constitue, de ce fait, un bon caractère discriminant. Elle est souvent utilisée pour discriminer les espèces (Puig et al., 2003; Metre et Ghorpade, 2013 ; Ngom et al., 2016).

- Le caractère qualitatif du limbe lié à sa sensation au toucher permet d'isoler 4 espèces 
étudiées dont 2 ( $F$. capreaefolia et $F$. exasperata) présentant une rugosité plus prononcée de la surface de leurs limbes. La sensation au toucher est également un caractère taxonomique important chez les espèces du genre Ficus (Diop, 2013; Nur Fatihal et al., 2014 ; Bosdeveix, 2016).

- L'utilisation de la marge du limbe comme caractère discriminant permet d'isoler un groupe restreint de 4 espèces à marge sinueuse ou dentée. Il s'agit de : F. exasperata, F. sur, F. sycomorus et $F$. vallis-choudae (Figure 7 ; Figure 19 ; Figure 20 et Figure 24). La marge sinueuse ou dentée est un caractère taxonomique assez commode pour différentier F. sur et F. vallis-choudae (Mat et al., 2012 ; Metre et Ghorpade, 2013). Elle est très souvent utilisée en taxonomie pour préciser l'identité des espèces (Gaaliche et al., 2012).

- Les dimensions (longueur et largeur) du limbe sont :

de bons caractères de groupes car elles permettent de séparer avec précision les espèces à limbe de petite taille des autres espèces à limbe de taille moyenne (Puig et al., 2003) ;

- souvent déterminantes dans la discrimination au plan morphologique des espèces affines telles que $F$. thonningii (Figure 21) et F. iteophylla (Figure 12).

Ces résultats confirment l'importance taxonomique des caractères qualitatifs et quantitatifs du limbe chez les espèces du genre Ficus. Cette importance taxonomique a été confirmée par les travaux de plusieurs auteurs pour le genre Ficus (Sonibare et al., 2004 ; Diop, 2013 ; Loutfy et al., 2005 ; Adeyemi et al., 2013 ; Dhungana et al., 2015 ; Iroka et al., 2015 et pour d'autres genres (Hargreaves, 2006 ; Iroka et al., 2015 ; Nwokeocha, 2015 ; Semde et al., 2015).

A l'échelle intraspécifique, les résultats obtenus montrent que les caractères étudiés ne permettent pas de discriminer les 2 variétés de $F$. glumosa au plan morphologique (Figures 8 et 9 ). Les caractères différentiels entre ces 2 variétés s'observent au niveau de la densité et de la diversité des formes de trichomes de leurs organes reproducteurs. Ces résultats sont importants car ils confirment le statut de variété de ces taxons très proches (Diop, 2013).

Au total, l'étude a permis de déceler des caractères discriminants pertinents qui ont permis d'identifier 20 sur les 24 espèces étudiées. Cependant la grande ressemblance morphologique entre les 2 variétés de glumosa et 2 espèces synonymes ( $F$. iteophylla et $F$. thonningii) exige que cette étude soit élargie à d'autres caractères pour une discrimination plus précise des espèces. Pour cette raison, une étude qualitative et quantitative des figues a été entreprise afin de préciser et de compléter les caractères morphologiques étudiés pour l'identification des espèces du genre Ficus L.

\section{Morphologie de l'appareil reproducteur Caractères qualitatifs des figues}

L'analyse des résultats (Tableau 3) montre que les caractères qualitatifs des figues permettent de caractériser individuellement la moitié des espèces étudiées. Il s'agit de $F$. conraui, $F$. cordata, $F$. ingens, $F$. lutea, $F$. ottoniifolia, F. ovata, F. polita, F. sur, $F$. sycomorus, $F$. thonningii, $F$. umbellata et $F$. vallis-choudae. Ces caractères qualitatifs ont une importance taxonomique réelle car ils sont fréquents et stables chez l'espèce (Zhekun and Gilbert, 2003 ; Kumar et al., 2011 ; Gaaliche, 2012).

Certains caractères tels que les éperons, les calyptres, la forme de l'ostiole et la position des fleurs mâles ne sont pas très souvent utilisés dans les clés d'identification par les auteurs (Puig et al. 2003 ; Diop, 2013). Les résultats obtenus montrent la pertinence du choix de ces caractères étudiés pour l'identification des espèces du genre Ficus. Ils apportent à la clé de la Flore du Sénégal de Berhaut (1967) un complément d'informations nécessaire à une identification précise des espèces du genre Ficus. Ces résultats confirment l'importance des 
caractères qualitatifs des organes reproducteurs dans la taxonomie des espèces (Shu, 2003 ; Oladipo et al., 2010 ; Kumar et al., 2011; Lansky et Paavilainen, 2017).

Pour compléter cette étude, des caractères morphométriques des figues sont analysés pour préciser davantage l'identité des différentes espèces étudiées.

\section{Caractères quantitatifs des figues}

L'analyse des résultats (Tableau 4) montre que la variabilité de la longueur des pédoncules des figues permet d'observer 3 groupes d'espèces de Ficus. Il s'agit d'espèces dont les figues sont :

- sessiles ou sans pédoncule ;

- courtement pédonculées ;

- nettement pédonculées.

Dans chaque groupe, la taille des figues permet de distinguer soit des espèces telles que $F$. conraui, $F$. lutea, $F$. ovata $F$. thonningii et $F$. vallis-choudae; soit des sousgroupes d'espèces.

Les caractères quantitatifs des figues sont souvent utilisés pour identifier les différentes espèces du genre Ficus et leur importance taxonomique a été attestée par plusieurs auteurs (Zhekun et Gilbert. 2003 ; Sonibare et al., 2004 ; Polat et Caliskan, 2008 ; Gaaliche et al., 2012 ; Lansky et Paavilainen, 2017).

Sur la base des caractères morphologiques et quantitatifs des feuilles et des figues, la clé de détermination suivante a été élaborée pour identifier les différentes espèces du genre Ficus.

1. Figues sessiles à subsessiles

2. Figues nettement sessiles ou dépourvues de pédoncules, ostiole en fente

3. Figues pubescentes, 1,5 à $1,6 \mathrm{~cm}$ de diamètre, feuille ovale... $F$. lutea

3'. Figues glabres

4. Feuille obovale à base aigue, figues de diamètre 1,9 à $2 \mathrm{~cm}$...F. conraui.

4'. Feuille elliptique à base arrondie, figues de diamètre 0,8 à $1 \mathrm{~cm}$.Présencemarquée de nombreuses racines adventives sur les grosses branches.. F. thonningii
2'. Figues subsessiles

5. Ostiole circulaire, bractéoles visibles de l'extérieur. Feuille ovale à elliptique

6. Figues de petites dimensions 0,8 à $1,10 \mathrm{~cm}$ de diamètre, par paire à l'aisselle des feuilles.

7. Figues pubescentes, présence d'éperons à la base des pédoncules. Port arborescent... F. ingens

7'. Figues glabre, absence d'éperons à la base des pédoncules. Port arbustif...F. cordata

6'. Figues de grandes dimensions 4 à $4,6 \mathrm{~cm}$

de diamètre, solitaires à l'aisselle des feuilles... F. vallis-choudae

5'. Ostiole en fente, bractéoles non visibles de l'extérieur, feuille ovale, présence de calyptres bien développés à l'aisselle des feuilles, éperons absents ...F. ovata

1'. Figues pédonculées

8. Feuilles entièrement ou partiellement scabres

9. Feuilles, figues et pétioles entièrement scabres. Présence de racines adventives

10. Port buissonnant, feuilles elliptiques non cordées, souvent opposées $F$. capreaefolia

10'. Port arbustif ou arborescent, feuilles ovales, alternes $F$. exasperata

9'. Figues et pétioles non scabres, face supérieure des feuilles légèrement scabres, Ostiole circulaire, bractéoles visibles de l'extérieur. Absence de racines adventives

11. Figues velues, longuement pédonculées $(\mathrm{Lp}=1,8 \mathrm{~cm})$, de grandes dimensions $(\mathrm{Dm}=$ $3,2 \mathrm{~cm}) \ldots F$. sycomorus

11.' Figues glabres, moyennement pédonculées $(\mathrm{Lp}=1,2 \mathrm{~cm})$ et de petites dimensions $(\mathrm{Dm}=1,2 \mathrm{~cm}) \ldots F$. dicranostyla

8. Feuilles non scabres, lisses

12. Figues de petite dimension $(\mathrm{Dm} \leq 1,3 \mathrm{~cm})$

13. Feuilles ovales

14. Feuilles de petite dimension

15. Figues glabres...F. glumosa var. glaberrima.

15'.Figues pubescentes...F. glumosa var. glumosa

14'. Feuilles de grande taille

16. Figues par paire à l'aisselle des pédoncules. Pédoncule long de $0,9 \mathrm{~cm}$...F. trichopoda 
16.' Figues en grappe à l'extrémité des rameaux. Pédoncule long de $1,4 \mathrm{~cm} \ldots F$. platyphylla

13'. Feuilles non ovales

17. Feuilles cordées à base arrondie, de forme suborbiculaire $F$. abutilifolia

17'. Feuilles non cordées à base aigue

18. Feuilleobtriangulaire...F. natalensis

18'.Feuille elliptique...F. iteophylla

12 '. Figues de dimensions moyennes $(\mathrm{Dm} \geq$ $1,4 \mathrm{~cm})$

19. Espèces cauliflores

20. Feuilles ovales

21. Figues en grappes sur des ramilles aphylles du tronc ou des grosses branches. Feuilles faiblement cordées à sommet obtus...F. sur

21 '.Figues en grappes sur des éperons des grosses

branches. Feuilles largement cordées à sommet

longuement acuminé...F. polita

20'. Feuilles suborbiculaires à elliptiques.

Présence d'éperons à

la base des pédoncules

22. Feuilles suborbiculaires à base cordée et à sommet

courtement acuminé $F$. umbellata

22'. Feuilles elliptiques à base aigue et à sommet longuement acuminé. F. ottoniifolia

19'. Espèces non cauliflores

23. Feuilles à base aigue et de dimension moyenne $($ Longueur $=15 \mathrm{~cm}$ et largeur $=13$ $\mathrm{cm})$. Pédoncule de long $1,5 \mathrm{~cm} \quad \ldots . . . F$. elasticoides

23'. Feuilles à base arrondie et de petite dimension $($ Longueur $=11 \mathrm{~cm}$ et largeur $=7,5$ $\mathrm{cm})$. Pédoncule long de $0,9 \mathrm{~cm} . . F$. scottelliotii

Cette clé montre l'importance de l'apport des caractères qualitatifs et quantitatifs des feuilles et des figues dans la détermination des espèces du genre Ficus L.

La forme caractéristique des figues (réceptacles urcéolés) a permis :

- de distinguer le genre Ficus des autres genres de la famille des Moraceae ;
- d'apporter plus de précision dans la taxonomie des espèces du genre Ficus $\mathrm{L}$.

Dans cette clé, l'absence ou la présence du pédoncule ainsi que la taille de la figue apparaissent comme étant des caractères importants en taxonomie car ils permettent de distinguer de façon précise trois groupes d'espèces : les espèces à figues sessiles, les espèces à figues subsessiles et les espèces à figues pédonculées. La pertinence de l'utilité de ces caractères dans le domaine de la taxonomie a été prouvée par les travaux de plusieurs auteurs (Polat et Caliskan, 2008 ; Kumar et al., 2011).

Comme pour le pédoncule, la forme de l'ostiole du réceptacle permet de séparer les espèces à ostiole circulaire des espèces à ostiole en fente. Sa stabilité chez l'espèce revêt toute l'importance qu'on lui accorde dans la systématique des espèces du genre Ficus L. La position des organes reproducteurs est un caractère souvent utilisé par les auteurs pour la détermination des espèces (Puig et al., 2003 ; Harrison et al., 2012). Elle a surtout permis de distinguer $F$. polita et $F$. sur des autres espèces étudiées. Ces espèces ( $F$. polita et $F$. sur) portent leurs figues, respectivement, sur leurs grosses branches uniquement et sur le tronc principalement.

La variation de la position des figues d'une espèce à une autre est un caractère taxonomique important chez le genre Ficus (Kumar et al., 2011). Elle permet surtout de caractériser les espèces cauliflores comme $F$. polita, F. umbellata, F. lutea et $F$. sur.

La sensation au toucher est aussi un caractère taxonomique important (Puig et al., 2003). Ce caractère permet de distinguer $F$. capreaefolia et $F$. exasperata des autres espèces. En effet, ces espèces sont les seules à posséder des figues rugueuses ou scabres. $\mathrm{Ce}$ caractère a été utilisé par Diop (2013) pour distinguer ces espèces des autres espèces rencontrées au Sénégal.

Les résultats ont montré que la présence ou l'absence de poils permet de séparer les espèces à figues pubescentes $(F$. 
glumosa var. glumosa, F. lutea, F. ingens) des autres espèces à figues glabres mais également de distinguer les deux variétés de $F$. glumosa. La pilosité est, de ce fait, importante pour caractériser les variétés de $F$. glumosa. Elle a été utilisée par Berhaut (1967) et Diop (2013) dans les clés de détermination des espèces du genre Ficus qu'ils ont proposées.

La présence d'un calyptre à l'aisselle des feuilles n'est observée que chez $F$. ovata (Figure 15). Il s'agit donc d'un caractère rare qui permet de distinguer cette espèce des autres. La présence d'un calyptre est souvent ignorée par les auteurs du fait de sa fugacité chez l'espèce. Toutefois, il demeure important en taxonomie pour le genre Ficus.

La présence très marquée des éperons à la base des pédoncules chez les espèces comme $F$. ottoniifolia, $F$. polita et $F$. umbellata (Figure14, Figure 17 et Figure 23) a permis de les distinguer des autres espèces étudiées. Ce caractère est absent des clés de la Flore du Sénégal. Les éperons ne sont observés que chez quelques espèces du genre Ficus et constituent, de ce fait, un bon caractère distinctif contrairement à l'avis de Berg et al. (1985) qui le juge peu fiable du fait de son irrégularité chez l'espèce.

Les caractères floraux ont été peu utilisés jusqu'à présent par rapport à ceux des feuilles. Toutefois, ils sont taxonomiquement très importants du fait de leur régularité chez l'espèce et de leur variabilité dans le genre Ficus L. La disposition des fleurs mâles dans la cavité intérieure du réceptacle a permis de distinguer les espèces à fleurs mâles localisées près de l'ostiole, des espèces à fleurs mâles disséminées entre les fleurs femelles (Zhekun and Gilbert, 2003).

La disposition des fleurs mâles dans la figue semble être liée à la forme de l'ostiole du réceptacle car toutes les espèces étudiées à ostiole circulaire ont leurs fleurs mâles situées près de l'ostiole. Ce caractère taxonomique important a été déjà utilisé par Berg et al. (1985) et par Zhekun and Gilbert (2003).

L'importance des caractères qualitatifs des organes végétatifs et reproducteurs dans les études systématiques des espèces végétales, en général (Knapp et Naczi, 2008 ; Iroka et al., 2015 ; Gbaguidi et al., 2015) et celles du genre Ficus L., en particulier, est incontestable (Clement and Weiblen, 2009; Diop, 2013). Du fait de leur diversité de forme, de taille et de sa position sur la plante, les réceptacles urcéolés ou figues sont très utilisés par les auteurs pour la détermination des espèces du genre Ficus (Harrison et al., 2012 ; Diop, 2013). De plus en plus, les caractères morphologiques des organes reproducteurs sont souvent à la base des clés d'identification chez les végétaux car ils sont plus stables au sein de l'espèce comparés aux caractères végétatifs très dépendants des conditions du milieu (Knapp et Naczi, 2008 ; Clement and Weiblen, 2009 ; Diop, 2013 ; Iroka et al., 2015 ; Gbaguidi et al., 2015). La clé d'identification des espèces du genre Ficus de la «Flore de Sénégal » de Berhaut (1967), qui est le document de référence actuel, utilise essentiellement à sa base les caractères de l'appareil végétatif pour toute identification. Cette étude, au contraire, propose une clé d'identification qui s'appuie à sa base sur les caractères morphologiques de l'appareil reproducteur (figues) puis associe secondairement les caractères morphologiques et morphométriques des organes végétatifs et reproducteurs pour identifier les différentes espèces du genre Ficus au Sénégal. La stabilité des caractères des figues et les illustrations proposées (Figures 1 à 24) pour chacune des espèces étudiées donnent plus de précision et de commodité pour l'identification des différents taxons du genre Ficus. 
Tableau 1 : Principaux caractères morphologiques de la plante adulte des espèces étudiées du genre Ficus L.

\begin{tabular}{|c|c|c|c|}
\hline \multirow[t]{2}{*}{ Espèces } & \multicolumn{3}{|c|}{ Caractères } \\
\hline & Type de port & Mode de développement & Racines aériennes \\
\hline F. abutilifolia & Arbustif (arbuste) & Non épiphyte & Absentes \\
\hline F. capreaefolia & Buissonnante & Non épiphyte & Présentes \\
\hline F. conraui & Arbustif & Hémi-épiphyte2 & Présentes \\
\hline F. cordata & Buissonnante & Non épiphyte & Présentes \\
\hline F. dicranostyla & Arbre & Non épiphyte & Absentes \\
\hline F. elasticoides & Arbre & Hémi-épiphyte & Présentes \\
\hline F. exasperata & Arbustif & Non épiphyte & Présentes \\
\hline F. glumosa var. glaberrima & Arbre & Non épiphyte & Présentes \\
\hline F. glumosa var. glumosa & Arbre & Non épiphyte & Présentes \\
\hline F. ingens & Arbre & Hémi-épiphyte & Présentes \\
\hline F. iteophylla & Arbre & Hémi-épiphyte & Présentes \\
\hline F. lutea & Arbre & Hémi-épiphyte & Présentes \\
\hline F. natalensis & Arbustif & Hémi-épiphyte & Présentes \\
\hline F. ottoniifolia & Arbustif & Hémi-épiphyte & Présentes \\
\hline F. ovata & Arbre & Hémi-épiphyte & Présentes \\
\hline F. platyphylla & Arbre & Non épiphyte & Présentes \\
\hline F. polita & Arbre & Hémi-épiphyte & Présentes \\
\hline F. scott-eliotii & Arbre & Hémi-épiphyte & Présentes \\
\hline F. sur & Arbustif & Non épiphyte & Absentes \\
\hline F. sycomorus & Arbre & Non épiphyte & Absentes \\
\hline F. thonningii & Arbre & Hémi-épiphyte & Présentes \\
\hline F. trichopoda & Arbre & Hémi-épiphyte & Présentes \\
\hline F. umbellata & Arbre & Hémi-épiphyte & Présentes \\
\hline F. vallis-choudae & Arbre & Non épiphyte & Présentes \\
\hline
\end{tabular}

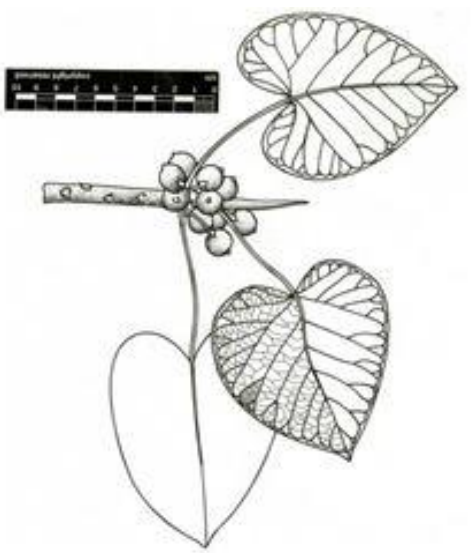

Figure 1: F. abutilifolia

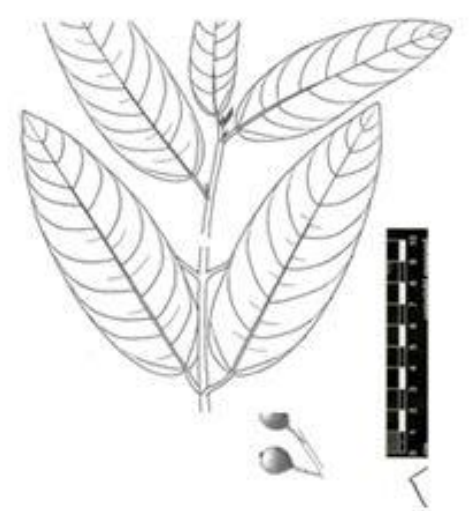

Figure 2: $F$. capreaefolia 
Tableau 2 : Caractères qualitatifs et quantitatifs de la feuille adulte des espèces du genre Ficus L.

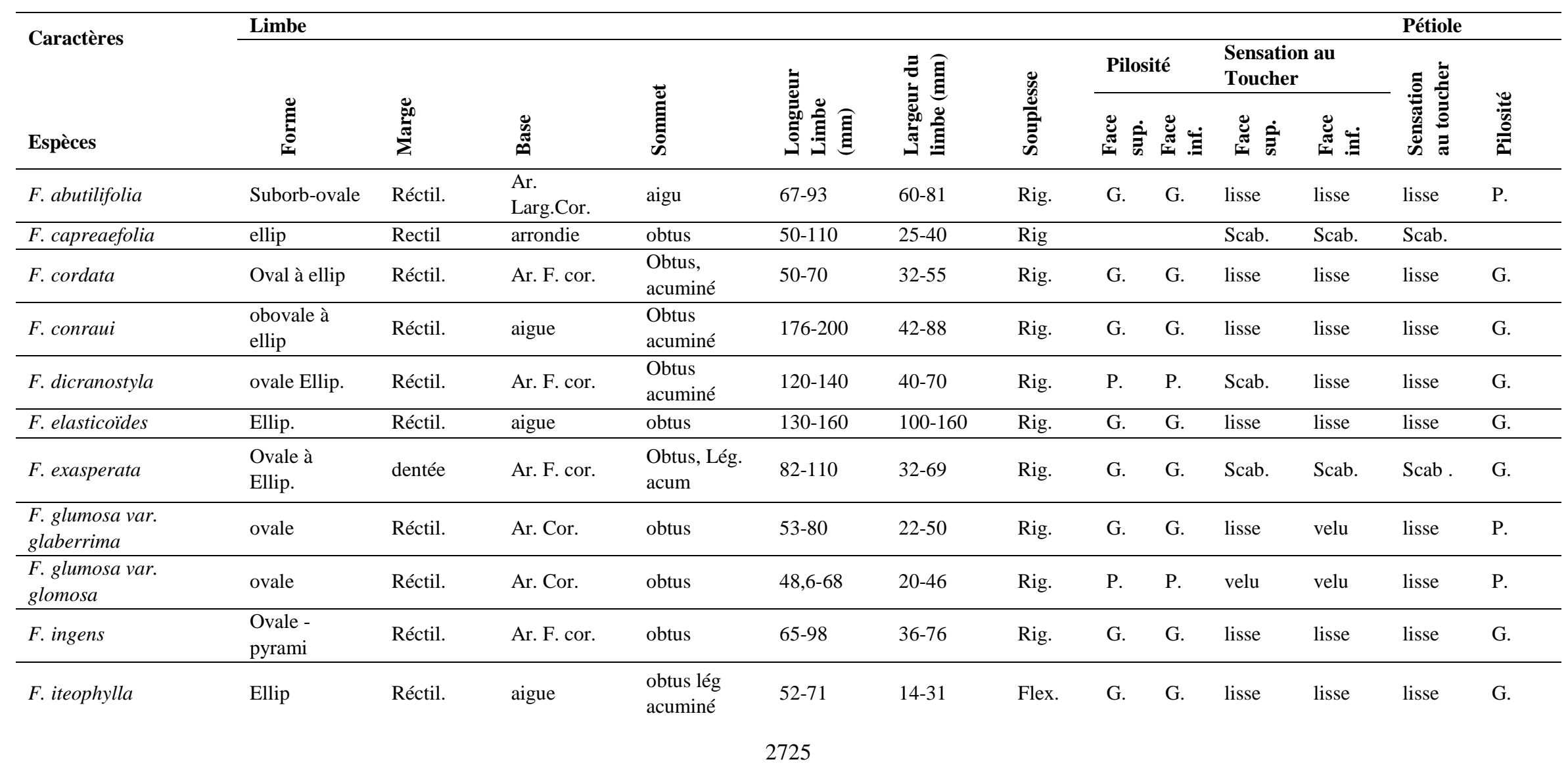


D. DIOP et al. / Int. J. Biol. Chem. Sci. 12(6): 2713-2737, 2018

\begin{tabular}{|c|c|c|c|c|c|c|c|c|c|c|c|c|c|}
\hline \multirow{3}{*}{$\begin{array}{l}\text { Caractères } \\
\text { Espèces }\end{array}$} & \multicolumn{7}{|l|}{ Limbe } & \multicolumn{6}{|c|}{ Pétiole } \\
\hline & \multirow[b]{2}{*}{ 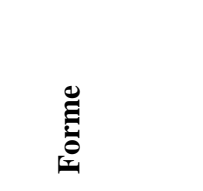 } & \multirow[b]{2}{*}{ 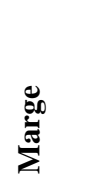 } & \multirow[b]{2}{*}{ 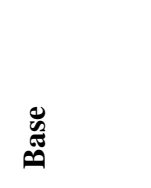 } & \multirow{2}{*}{ 总 } & \multirow{2}{*}{ 总 } & \multirow{2}{*}{ 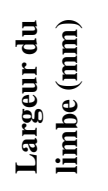 } & \multirow{2}{*}{ 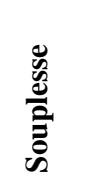 } & \multicolumn{2}{|c|}{ Pilosité } & \multicolumn{2}{|c|}{$\begin{array}{l}\text { Sensation au } \\
\text { Toucher }\end{array}$} & \multirow{2}{*}{ 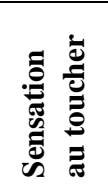 } & \multirow{2}{*}{ 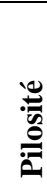 } \\
\hline & & & & & & & & 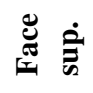 & 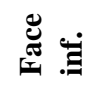 & 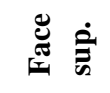 & 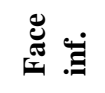 & & \\
\hline F. lutea & Ellip-ovale & Réctil. & Ar. & obtus & $170-190$ & $73-90$ & Rig. & G. & G. & lisse & lisse & lisse & G. \\
\hline F. natalensis & obtriangulaire & Réctil. & aigue & aplati & $49-77$ & $18-60$ & Rig. & G. & G. & lisse & lisse & lisse & G. \\
\hline F. ovata & ovale & Réctil. & Ar. F. cor. & $\begin{array}{l}\text { Obtus, } \\
\text { acuminé }\end{array}$ & $178-210$ & $89-110$ & Rig. & G. & G. & lisse & lisse & lisse & G. \\
\hline F. ottoniifolia & Ellip. & Réctil. & aigue & $\begin{array}{l}\text { Aigu, Long. } \\
\text { Acu. }\end{array}$ & $115-160$ & $57-77$ & Flex. & G. & G. & lisse & lisse & lisse & G. \\
\hline F. platyphylla & ovale & Réctil. & Ar. Cor. & obtus & $180-230$ & $94-155$ & Rig. & P. & $\mathrm{P}$ & lisse & lisse & lisse & G. \\
\hline F. polita & ovale & Réctil. & $\begin{array}{l}\text { Ar. } \\
\text { Larg.Cor. }\end{array}$ & $\begin{array}{l}\text { Obtus, Long. } \\
\text { acuminé }\end{array}$ & $152-215$ & $64-101$ & Flex. & G. & G. & lisse & lisse & lisse & G. \\
\hline F. scott-eliotii & Ellip. & Réctil. & Ar. & $\begin{array}{l}\text { Obtus, lég } \\
\text { acuminé }\end{array}$ & $106-118$ & $63-75$ & Rig. & G. & G. & lisse & lisse & lisse & G. \\
\hline F. sur & ovale Ellip & dentée & Ar. F. cor. & obtus & $119-160$ & $50-100$ & Flex. & G. & G. & lisse & lisse & lisse & G. \\
\hline F. sycomorus & $\begin{array}{l}\text { ovale - } \\
\text { suborbiculaire }\end{array}$ & $\begin{array}{l}\text { Lég. } \\
\text { dentée }\end{array}$ & $\begin{array}{l}\text { Ar. } \\
\text { Larg.Cor. }\end{array}$ & obtus & $122-156$ & $87-135$ & Rig. & G. & P. & Scab. & lisse & lisse & P. \\
\hline F. thonningii & Ellip. à Ovale & Réctil. & Ar. F. cor. & $\begin{array}{l}\text { obtus lég } \\
\text { acuminé }\end{array}$ & $52-89$ & $37-70$ & Flex. & G. & G. & lisse & lisse & lisse & G. \\
\hline F. trichopoda & ovale & Réctil. & $\begin{array}{l}\text { Ar. } \\
\text { Larg.Cor. }\end{array}$ & obtus & $\begin{array}{l}118-147 \\
2726\end{array}$ & $45-118$ & Rig. & G. & G. & lisse & lisse & lisse & G. \\
\hline
\end{tabular}


D. DIOP et al. / Int. J. Biol. Chem. Sci. 12(6): 2713-2737, 2018

\begin{tabular}{|c|c|c|c|c|c|c|c|c|c|c|c|c|c|}
\hline \multirow{3}{*}{$\begin{array}{l}\text { Caractères } \\
\text { Espèces }\end{array}$} & \multicolumn{7}{|l|}{ Limbe } & & & & \multicolumn{3}{|c|}{ Pétiole } \\
\hline & \multirow[b]{2}{*}{$\underset{\Xi}{\mathscr{0}}$} & \multirow[b]{2}{*}{ 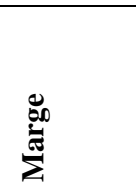 } & \multirow[b]{2}{*}{ 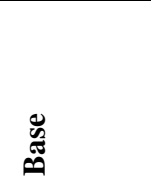 } & \multirow{2}{*}{$\underset{\mathscr{E}}{\stackrel{\Xi}{\Xi}}$} & \multirow{2}{*}{ 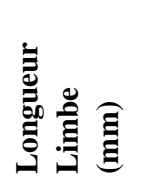 } & \multirow{2}{*}{ 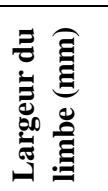 } & \multirow{2}{*}{$\frac{\tilde{n}}{\tilde{O}}$} & \multicolumn{2}{|c|}{ Pilosité } & \multicolumn{2}{|c|}{$\begin{array}{l}\text { Sensation au } \\
\text { Toucher }\end{array}$} & \multirow{2}{*}{ 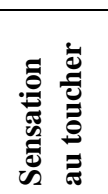 } & \multirow[b]{2}{*}{$\frac{\stackrel{2}{0}}{0}$} \\
\hline & & & & & & & & $\underset{\mathscr{E}}{\stackrel{\Xi}{\Xi}}$ & 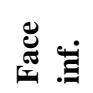 & $\underset{\tilde{\Xi}}{\stackrel{\Xi}{二}}$ & 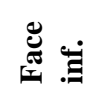 & & \\
\hline F. umbellata & suborbiculaire & Réctil. & $\begin{array}{l}\text { Ar. } \\
\text { Larg.Cor. }\end{array}$ & $\begin{array}{l}\text { Obtus Court. } \\
\text { acu. }\end{array}$ & $173-230$ & $65-101$ & Flex. & G. & G. & lisse & lisse & lisse & G. \\
\hline F. vallis-choudae & ovale & $\begin{array}{l}\text { sinueuse } \\
2 / 3 \text { sup. }\end{array}$ & Ar. Cor. & obtus & $119-160$ & $70-180$ & Flex. & G. & G. & lisse & lisse & lisse & G. \\
\hline
\end{tabular}

Ar. : Arrondie ; Cor. : Cordée ; Court. : courtement ; Ellip. : elliptique ; Flex. : Flexible ; G. : Glabr ; Lég. : légèrement

Long. : longuement ; P. : pubescent ; Rectil. : Rectiligne ; Rig. : rigide ; Scab. : Scabr ; Larg.= largeur ; Pyrami= pyramidale 
Tableau 3 : Caractères morphologiques des figues des espèces du genre Ficus L.

\begin{tabular}{|c|c|c|c|c|c|c|c|c|c|c|c|}
\hline $\begin{array}{l}\text { Caractères } \\
\text { étudiés } \\
\text { Espèces }\end{array}$ & $\begin{array}{l}\text { Localisation } \\
\text { des figues }\end{array}$ & Eperons & $\begin{array}{l}\text { Disposition } \\
\text { des figues }\end{array}$ & $\begin{array}{l}\text { Forme des } \\
\text { figues }\end{array}$ & Pédoncule & $\begin{array}{l}\text { Nbr. } \\
\text { bractée } \\
\text { basilaire }\end{array}$ & $\begin{array}{l}\text { Forme } \\
\text { de } \\
\text { l'ostiole }\end{array}$ & $\begin{array}{l}\text { Position } \\
\text { fleurs } \\
\text { mâles }\end{array}$ & $\begin{array}{l}\text { Pilosité/ } \\
\text { sensation } \\
\text { au } \\
\text { toucher }\end{array}$ & $\begin{array}{l}\text { Bractéoles } \\
\text { ostiolaires }\end{array}$ & $\begin{array}{l}\text { Calyptre } \\
\text { à } \\
\text { l'aisselle } \\
\text { des } \\
\text { feuilles }\end{array}$ \\
\hline F. abutilifolia & $\begin{array}{l}\text { Rameaux } \\
\text { feuillés }\end{array}$ & Absents & $\begin{array}{l}\text { En grappe à } \\
\text { l'extrémité } \\
\text { des } \\
\text { rameaux }\end{array}$ & Sphériques & Pédonculé & 2 & Fente & Disséminée & $\begin{array}{l}\text { Glabre / } \\
\text { Lisse }\end{array}$ & $\begin{array}{l}\text { Non } \\
\text { visibles }\end{array}$ & $\begin{array}{l}\text { Fugace, } \\
\text { peu } \\
\text { développé }\end{array}$ \\
\hline $\begin{array}{l}F . \\
\text { capreaefolia }\end{array}$ & $\begin{array}{l}\text { Rameaux } \\
\text { feuillés }\end{array}$ & Absents & $\begin{array}{l}\text { Rameaux } \\
\text { feuillés }\end{array}$ & Sphériques & Pédonculé & & Fente & Disséminée & $\begin{array}{l}\text { Glabre / } \\
\text { Scabre }\end{array}$ & $\begin{array}{l}\text { Non } \\
\text { visibles }\end{array}$ & $\begin{array}{l}\text { Fugace, } \\
\text { peu } \\
\text { développé }\end{array}$ \\
\hline F. conraui. & $\begin{array}{l}\text { Rameaux } \\
\text { feuillés }\end{array}$ & Absents & $\begin{array}{l}\text { Paire à } \\
\text { l'aisselle } \\
\text { des feuilles }\end{array}$ & Sphériques & Sessile & 2 & Fente & Disséminée & $\begin{array}{l}\text { Glabre / } \\
\text { Lisse }\end{array}$ & $\begin{array}{l}\text { Non } \\
\text { visibles }\end{array}$ & $\begin{array}{l}\text { Fugace, } \\
\text { peu } \\
\text { développé }\end{array}$ \\
\hline F. cordata & $\begin{array}{l}\text { Rameaux } \\
\text { feuillés }\end{array}$ & Absents & $\begin{array}{l}\text { Paire à } \\
\text { l'aisselle } \\
\text { des feuilles }\end{array}$ & Sphériques & Subsessile & 3 & Circulaire & $\begin{array}{l}\text { Près de } \\
\text { l'ostiole }\end{array}$ & $\begin{array}{l}\text { Glabre / } \\
\text { Lisse }\end{array}$ & visibles & $\begin{array}{l}\text { Fugace, } \\
\text { peu } \\
\text { développé }\end{array}$ \\
\hline F.dicranostyla & $\begin{array}{l}\text { Rameaux } \\
\text { feuillés }\end{array}$ & Absents & $\begin{array}{l}\text { Paire à } \\
\text { l'aisselle } \\
\text { des feuilles }\end{array}$ & $\begin{array}{l}\text { Sphériques, } \\
\text { peu aplati } \\
\text { au somme }\end{array}$ & Pédonculé & 3 & Circulaire & $\begin{array}{l}\text { Près de } \\
\text { l'ostiole }\end{array}$ & $\begin{array}{l}\text { Glabre / } \\
\text { Lisse }\end{array}$ & visibles & $\begin{array}{l}\text { Fugace, } \\
\text { peu } \\
\text { développé }\end{array}$ \\
\hline F.elasticoüdes & $\begin{array}{l}\text { Rameaux } \\
\text { feuillés }\end{array}$ & Absents & $\begin{array}{l}\text { Paire à } \\
\text { l'aisselle } \\
\text { des feuilles }\end{array}$ & Sphérique & Pédonculé & 2 & Fente & Disséminées & $\begin{array}{l}\text { Glabre / } \\
\text { Lisse }\end{array}$ & $\begin{array}{l}\text { Non } \\
\text { visibles }\end{array}$ & $\begin{array}{l}\text { Fugace, } \\
\text { peu } \\
\text { développé }\end{array}$ \\
\hline F. exasperata & Rameaux & Absents & Rameaux & Sphérique & $\begin{array}{r}\text { Pédonculé } \\
2728\end{array}$ & 3 & Circulaire & Près de & Glabre / & visibles & Fugace, \\
\hline
\end{tabular}




\begin{tabular}{|c|c|c|c|c|c|c|c|c|c|c|c|}
\hline & $\begin{array}{l}\text { feuillés } \\
\text { et grosses } \\
\text { branches }\end{array}$ & & feuillés & & & & & l'ostiole & Scabre & & $\begin{array}{l}\text { peu } \\
\text { développé }\end{array}$ \\
\hline $\begin{array}{l}\text { F. glumosa } \\
\text { var. } \\
\text { glaberrima }\end{array}$ & $\begin{array}{l}\text { Rameaux } \\
\text { feuillés } \\
\text { et grosses } \\
\text { branches }\end{array}$ & Absents & $\begin{array}{l}\text { Rameaux et } \\
\text { grosses } \\
\text { branches }\end{array}$ & Sphérique & Pédonculé & 2 & Fente & Disséminée & $\begin{array}{l}\text { Glabre / } \\
\text { Lisse }\end{array}$ & $\begin{array}{l}\text { Non } \\
\text { visibles }\end{array}$ & $\begin{array}{l}\text { Fugace et } \\
\text { peu } \\
\text { développé }\end{array}$ \\
\hline $\begin{array}{l}\text { F. glumosa } \\
\text { var. glumosa }\end{array}$ & $\begin{array}{l}\text { Rameaux } \\
\text { feuillés } \\
\text { et grosses } \\
\text { branches }\end{array}$ & Absents & $\begin{array}{l}\text { Rameaux et } \\
\text { grosses } \\
\text { branches }\end{array}$ & Sphérique & Pédonculé & 2 & Fente & Disséminée & $\begin{array}{l}\text { Pubescent } \\
\text { /Lisse }\end{array}$ & $\begin{array}{l}\text { Non } \\
\text { visibles }\end{array}$ & $\begin{array}{l}\text { Fugace, } \\
\text { peu } \\
\text { développé }\end{array}$ \\
\hline F.ingens & $\begin{array}{l}\text { Rameaux } \\
\text { feuillés }\end{array}$ & Presents & $\begin{array}{l}\text { Rameaux } \\
\text { feuillés }\end{array}$ & $\begin{array}{l}\text { Sphérique, } \\
\text { peu aplatis } \\
\text { au sommet }\end{array}$ & Pédonculés & 3 & Circulaire & $\begin{array}{l}\text { Près de } \\
\text { l'ostiole }\end{array}$ & $\begin{array}{l}\text { Pubescent } \\
\text { /Lisse }\end{array}$ & visibles & $\begin{array}{l}\text { Fugace, } \\
\text { peu } \\
\text { développé }\end{array}$ \\
\hline F. iteophylla & $\begin{array}{l}\text { Rameaux } \\
\text { feuillés }\end{array}$ & Absents & $\begin{array}{l}\text { Rameaux } \\
\text { feuillés }\end{array}$ & Sphérique & Pédonculé & 3 & Fente & Disséminée & $\begin{array}{l}\text { Pubescent } \\
\text { /Lisse }\end{array}$ & $\begin{array}{l}\text { Non } \\
\text { visibles }\end{array}$ & $\begin{array}{l}\text { Fugace, } \\
\text { peu } \\
\text { développé }\end{array}$ \\
\hline F. lutea & $\begin{array}{l}\text { Rameaux } \\
\text { feuillés } \\
\text { et grosses } \\
\text { branches }\end{array}$ & Absents & $\begin{array}{l}\text { Rameaux et } \\
\text { grosses } \\
\text { branches }\end{array}$ & Sphérique & Sessile & 2 & Fente & Disséminée & $\begin{array}{l}\text { Pubescent } \\
\text { /Lisse }\end{array}$ & $\begin{array}{l}\text { Non } \\
\text { visibles }\end{array}$ & $\begin{array}{l}\text { Fugace, } \\
\text { peu } \\
\text { développé }\end{array}$ \\
\hline F. natalensis & $\begin{array}{l}\text { Rameaux } \\
\text { feuillés }\end{array}$ & Absents & $\begin{array}{l}\text { Paire à } \\
\text { l'aisselle } \\
\text { des feuilles }\end{array}$ & Sphériques & Pédonculé & 2 & Fente & Disséminée & $\begin{array}{l}\text { Glabre / } \\
\text { Lisse }\end{array}$ & $\begin{array}{l}\text { Non } \\
\text { visibles }\end{array}$ & $\begin{array}{l}\text { Fugace, } \\
\text { peu } \\
\text { développé }\end{array}$ \\
\hline
\end{tabular}


Tableau 4 : Dimensions moyennes des figues [longueur moyenne (L), diamètre (D), longueur du pédoncule (Lp.)].

\begin{tabular}{|c|c|c|c|c|}
\hline $\mathbf{N}^{\circ}$ & Espèces & Lp en mm & L en mm & D en mm \\
\hline 1 & F. abutilifolia & $12,05^{\mathrm{f}}( \pm 0,83)$ & $14,25^{\mathrm{ij} 1}( \pm 1,32)$ & $12,88^{\text {eh }}( \pm 1,51)$ \\
\hline & F. capreaefolia & $12,07( \pm 0,32)$ & $13(+1,43)$ & $12(0,12)$ \\
\hline 2 & $F$. conraui & $0^{\mathrm{p}}$ & $20,13^{\mathrm{h}}( \pm 1,32)$ & $19,13^{\mathrm{e}}( \pm 1,53)$ \\
\hline 3 & F. cordata & $2,95^{\mathrm{p}}( \pm 0,82)$ & $8,47^{\mathrm{irt}}( \pm 1,31)$ & $9,18^{\mathrm{ej}}( \pm 1,51)$ \\
\hline 4 & F. dicranostyla & $12,08^{\mathrm{hij}}( \pm 0,83)$ & $13,74^{\mathrm{ijk}}( \pm 1,33)$ & $12,61^{\mathrm{ef}}( \pm 1,53)$ \\
\hline 5 & F.elasticoides & $14,5^{\mathrm{hi}}( \pm 0,82)$ & $16,5^{\mathrm{imo}}( \pm 1,33)$ & $17,5^{\mathrm{eh}}( \pm 1,52)$ \\
\hline 6 & F. exasperata & $9,5^{\mathrm{hi}}( \pm 0,83)$ & $11,47^{\mathrm{imq}}( \pm 1,32)$ & $12,12^{\text {ehi }}( \pm 1,53)$ \\
\hline 7 & F. glumosa var. glaberrima & $6,5^{\mathrm{hmn}}( \pm 0,84)$ & $10,12^{\text {inopq }}( \pm 1,33)$ & $9,58^{\mathrm{ei}}( \pm 1,51)$ \\
\hline 8 & F. glumosa var. glumosa & $8,67^{\mathrm{hmn}}( \pm 0,83)$ & $10^{\text {inopqr }}( \pm 1,31)$ & $11,5^{\text {eh }}( \pm 1,52)$ \\
\hline 9 & F. iteophylla & $9,58^{\text {hik }}( \pm 0,82)$ & $7,33^{\text {it }}( \pm 1,31)$ & $6,42^{\mathrm{el}}( \pm 1,53)$ \\
\hline 10 & F. ingens & $4,96^{\mathrm{hj}}( \pm 0,83)$ & $10,93^{\mathrm{imn}}( \pm 1,32)$ & $11,09^{\mathrm{efg}}( \pm 1,51)$ \\
\hline 11 & F. lutea & $0^{\mathrm{p}}$ & $16,03^{\mathrm{ij}}( \pm 1,33)$ & $15,24^{\text {efg }}( \pm 1,53)$ \\
\hline 12 & F. natalensis & $9,93^{\mathrm{g}}( \pm 0,82)$ & $9,90^{\text {inopqr }}( \pm 1,32)$ & $9,05^{\mathrm{ek}}( \pm 1,53)$ \\
\hline 13 & F. ottoniifolia & $15,44^{\mathrm{ce}}( \pm 0,82)$ & $15,85 b g( \pm 1,31)$ & $12,35^{\mathrm{ef}}( \pm 1,53)$ \\
\hline 14 & F. ovata & $3,05^{\mathrm{h} k \mathrm{~lm}}( \pm 0,82)$ & $32,13^{\text {bcd }}( \pm 1,32)$ & $27,85^{\mathrm{bc}}( \pm 1,52)$ \\
\hline 15 & F. platyphylla & $14,90^{\mathrm{cd}}( \pm 0,83)$ & $12,65^{\mathrm{iklm}}( \pm 1,32)$ & $12,46^{\mathrm{egh}}( \pm 1,52)$ \\
\hline 16 & $F$. polita & $19,69^{\mathrm{b}}( \pm 0,83)$ & $27,74^{\text {bef }}( \pm 1,31)$ & $29,62^{\mathrm{bc}}( \pm 1,52)$ \\
\hline 17 & F. scott - eliotii & $8,61^{\mathrm{hjl}}( \pm 0,82)$ & $29,17^{\text {bde }}( \pm 1,31)$ & $30,94^{\mathrm{bc}}( \pm 1,51)$ \\
\hline 18 & F. sur & $15,68^{\text {cde }}( \pm 0,83)$ & $24,47^{\mathrm{bg}}( \pm 1,32)$ & $23,17^{\mathrm{d}}( \pm 1,52)$ \\
\hline 19 & F. sycomorus & $18,77^{\mathrm{a}}( \pm 0,83)$ & $26,12^{\mathrm{bc}}( \pm 1,32)$ & $32,07^{\mathrm{b}}( \pm 1,51)$ \\
\hline 20 & F. thonningii & $0^{\mathrm{p}}$ & $8,40^{\text {irt }}( \pm 1,33)$ & $9,54^{\mathrm{ekl}}( \pm 1,51)$ \\
\hline & F. trichopoda & $9^{g}( \pm 0,82)$ & $10,85^{\text {imp }}( \pm 1,32)$ & $11,73^{\text {ehj }}( \pm 1,51)$ \\
\hline 22 & F. umbellata & $9,90^{\mathrm{hm}}( \pm 0,52)$ & $25,05^{\mathrm{bfg}}( \pm 1,32)$ & $23,42^{\mathrm{d}}( \pm 1,52)$ \\
\hline & F. vallis- choudae & $3,80^{\mathrm{hkTm}}( \pm 0,83)$ & $40,68^{\mathrm{a}}( \pm 1,22)$ & $46,23^{\mathrm{a}}( \pm 1,43)$ \\
\hline
\end{tabular}

NB : - Entre les parenthèses : intervalle de confiance de la moyenne, La première lettre en exposant de la moyenne du variable considéré indique le groupe d'appartenance c'est-à-dire que les moyennes de ce groupe sont comparables au point de vue statistique. Les lettres suivantes indiquent les sous-groupes d'appartenance c'est-à-dire que les moyennes portant ces lettres sont comparables en valeur absolue. Le seuil de significativité est fixé à $5 \%(\mathrm{p}<0.05)$. 


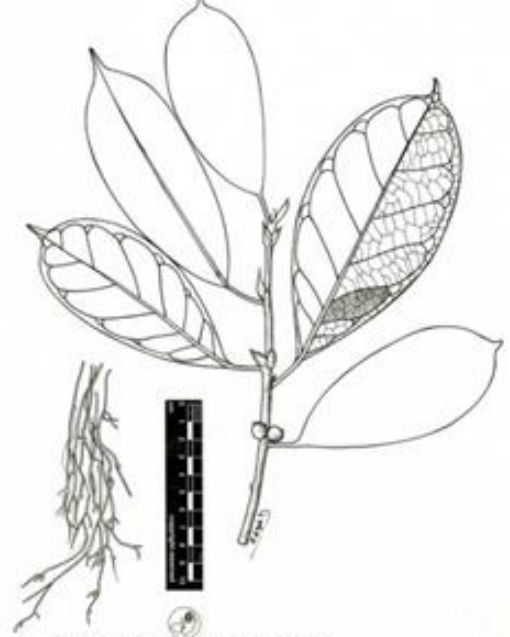

Figure 3: F. conraui

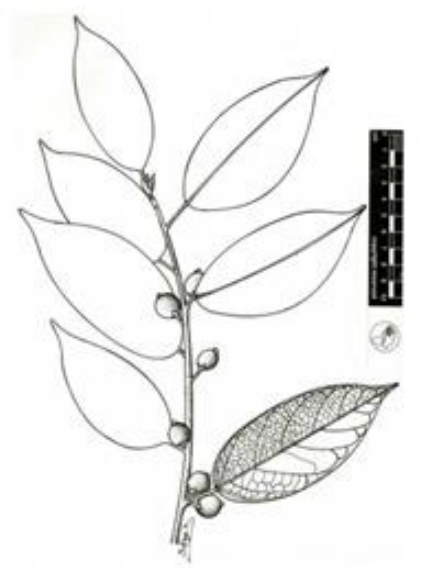

Figure 5: F. dicranostylla

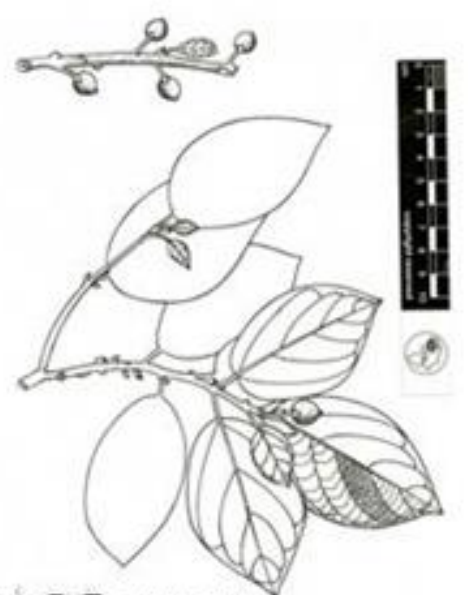

Figure 7: F. exasperata

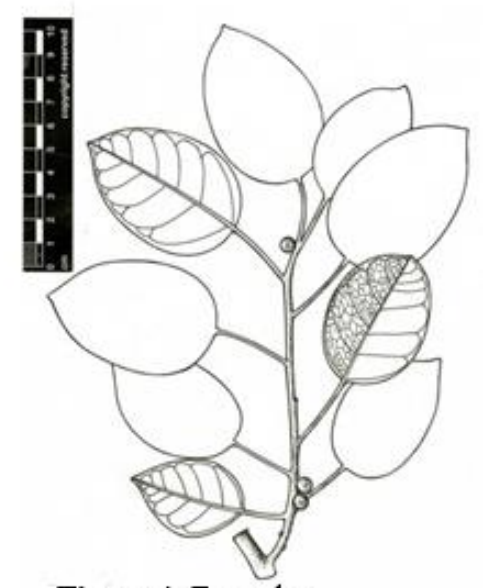

Figure 4: F. cordata

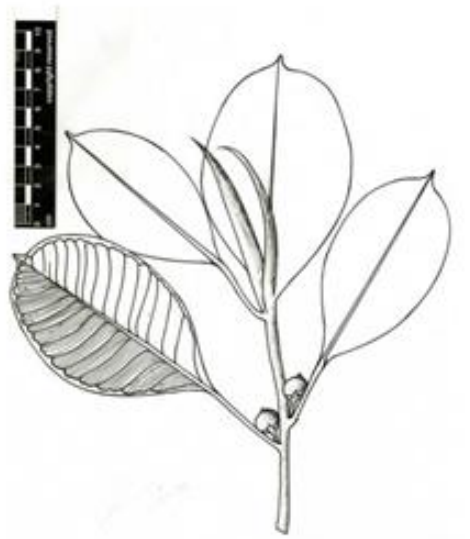

Figure 6: F. elasticoides

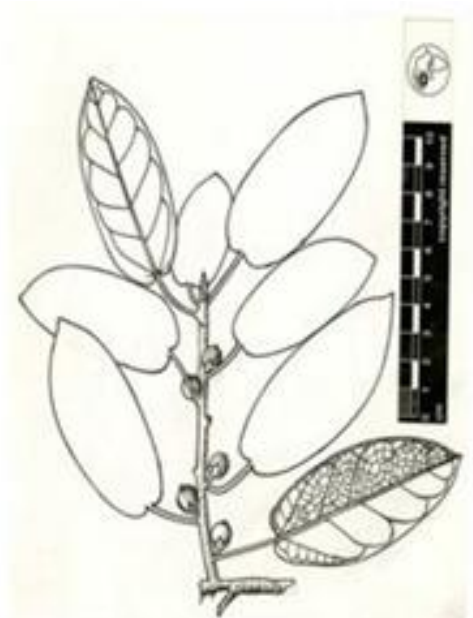

Figure 8: F. glumosa var. glaberrima 


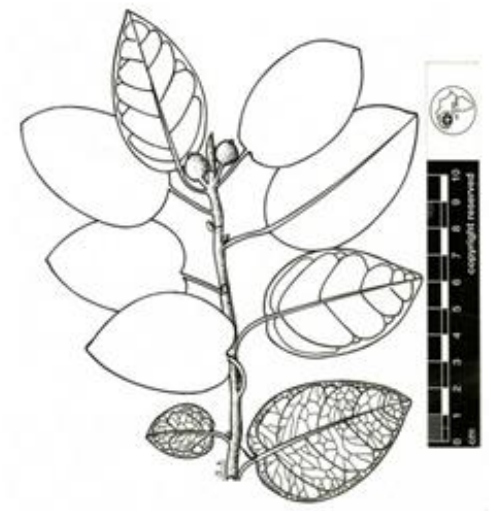

Figure 9: F. glumosa var. glumosa

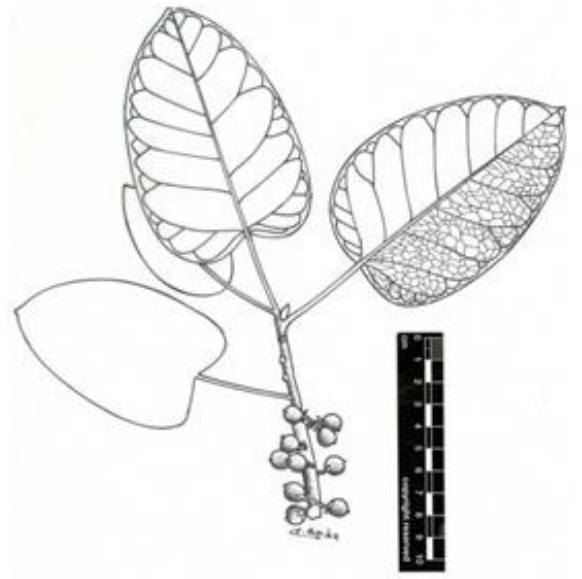

Figure 10: $F$. ingens

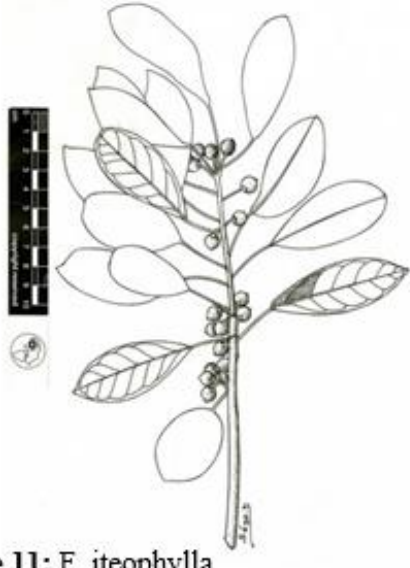

Figure 11: F. iteophylla

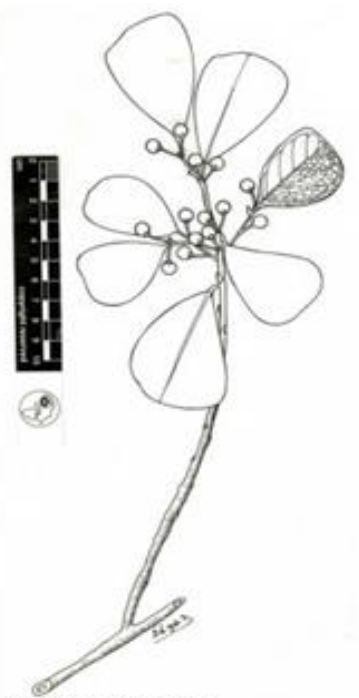

Figure 13: F. natalensis

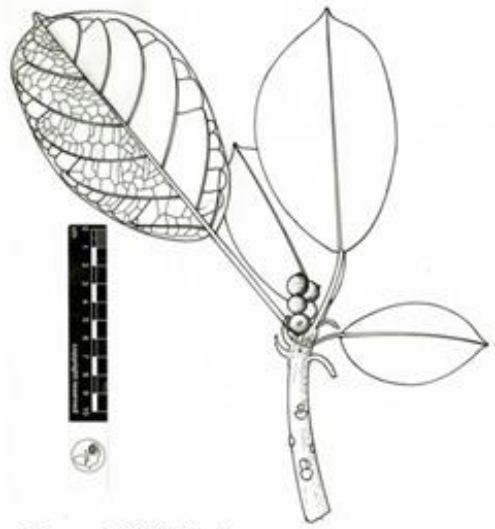

Figure 12: F. lutea

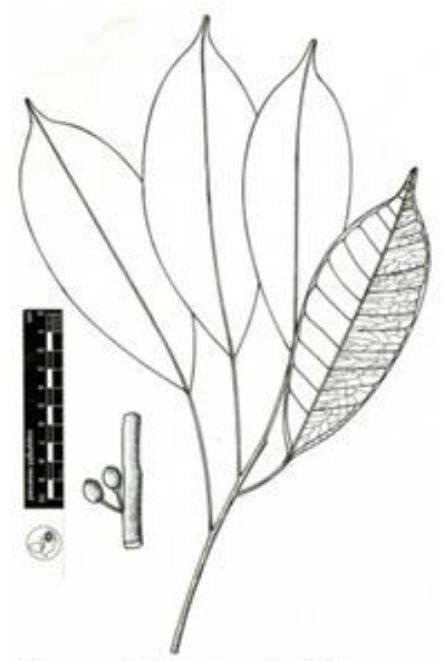

Figure 14: F. ottoniafolia 
D. DIOP et al. / Int. J. Biol. Chem. Sci. 12(6): 2713-2737, 2018

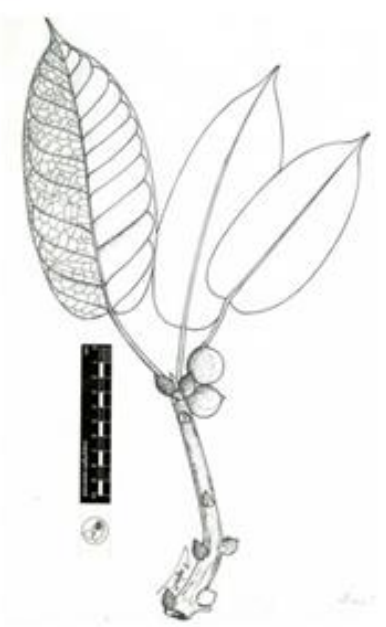

Figure 15: F. ovata

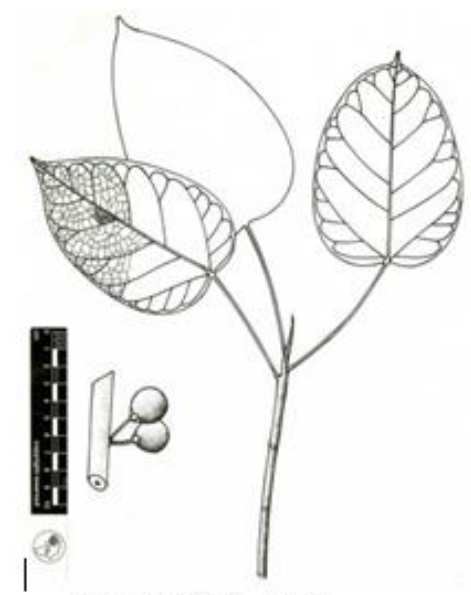

Figure 17: F. polita

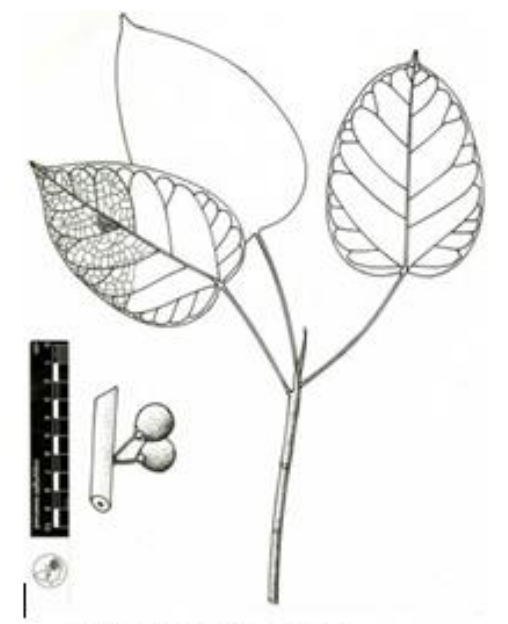

Figure 17: F. polita

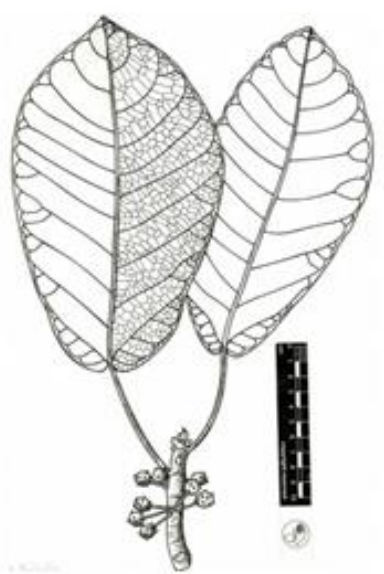

Figure 16: F. platyphylla

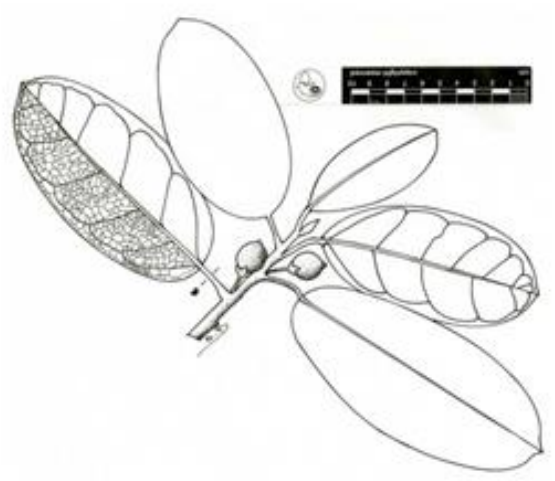

Figure 18: F. scott-ellioti

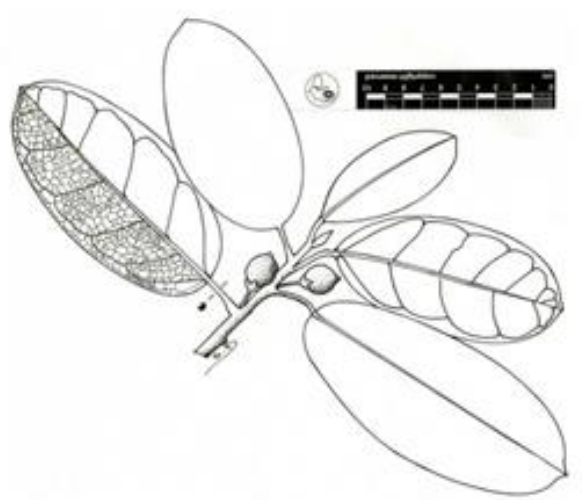

Figure 18: F. scott-ellioti 
D. DIOP et al. / Int. J. Biol. Chem. Sci. 12(6): 2713-2737, 2018

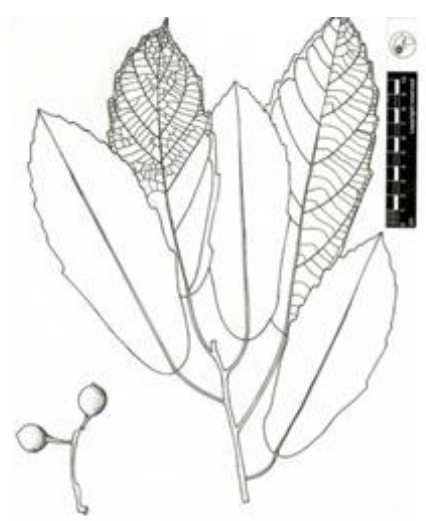

Figure 19: F. sur

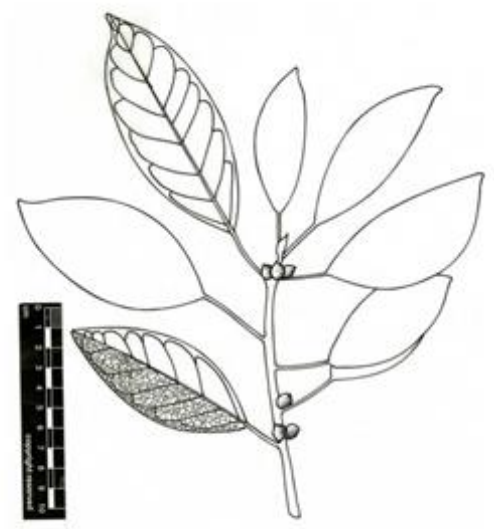

Figure 21: $F$. thonningii

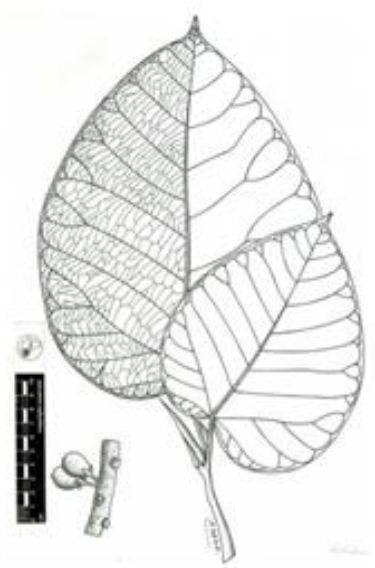

Figure 23: F. umbellata

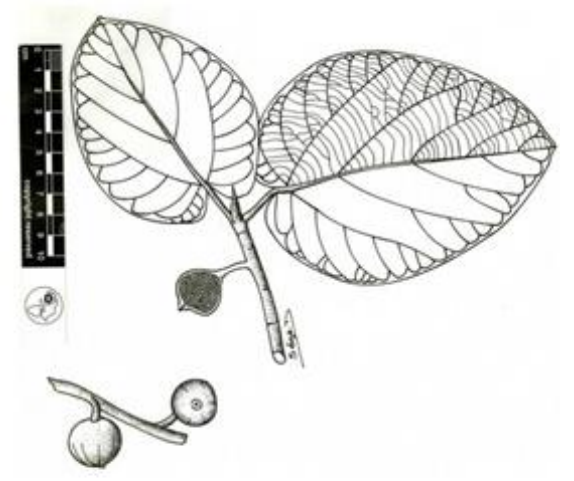

Figure 20: F. sycomorus

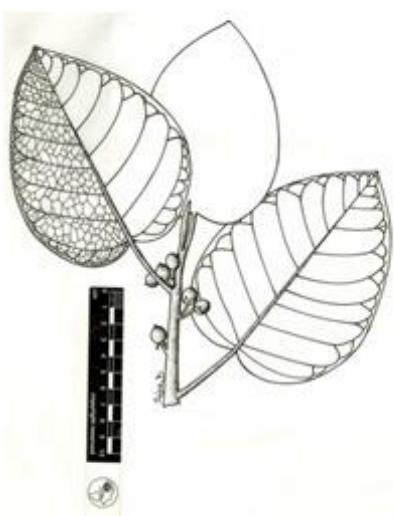

Figure 22: F. trichopoda

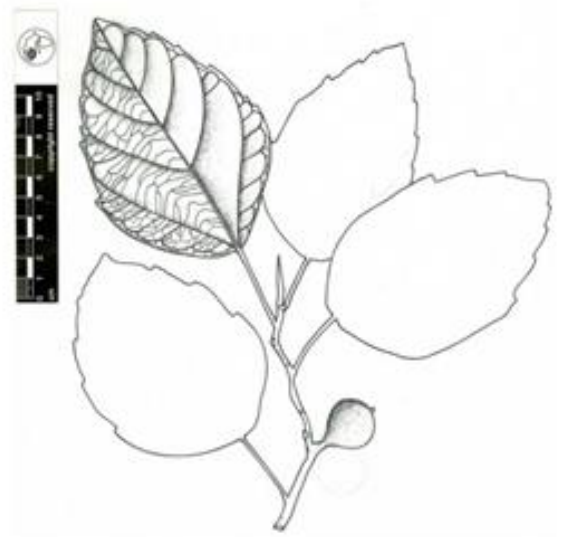

Figure 24 : $\mathrm{E}$. vallis-choudae 


\section{Conclusion}

L'objectif visé par cette étude est de montrer l'apport taxonomique des caractères morphologiques chez le genre Ficus et de proposer une clé d'identification des espèces rencontrées au Sénégal. L'étude a permis de revisiter les caractères systématiques des organes végétatifs et reproducteurs qui ont contribué de façon pertinente à l'identification des différentes espèces étudiées. Ces caractères morphologiques, quantitatifs et qualitatifs des appareils végétatif et reproducteur ont permis la caractérisation de différentes unités taxonomiques mais également l'identification de toutes les espèces et variétés du genre Ficus au Sénégal. Ces résultats confortent l'importance taxonomique des caractères morphologiques chez le genre Ficus. Ils permettent de proposer une clé d'identification commode et précise pour les différents taxa étudiés. Les illustrations des espèces dans cette étude rendent cette clé plus pratique.

\section{CONFLIT D'INTERETS}

Les auteurs déclarent qu'il n'y a aucun conflit d'intérêts pour ce manuscrit.

\section{CONTRIBUTIONS DES AUTEURS}

MSM : En mission de terrain avec l'auteur principal de l'article pour prospecter, collecter et identifier les différents échantillons; SS a contribué aux mesures et à l'analyse statistique des données ; KN a participé à la conception du travail et à la rédaction de cet article; ATB a apporté une contribution importante sur le fond et la forme de ce travail.

\section{RÉFÉRENCES}

Abdi H, Williams LJ. 2010. Newman-Keuls test and Tukey test. In: Salkind, N. (Ed.), Encyclopedia of Research Design, Thousand Oaks, pp. 1-11. https://www.utdallas.edu/ herve/abdiNewmanKeuls2010-pretty.pdf

Adeyemi TO, Ogundipe OT, Olowokudejo JD. 2013. A review of the taxonomy of African Sapindaceae based on quantitative and qualitative characters. Ife Journal of Science, 15(2): 303-314. https://www.ajol.info/index.php/ijs/artic le/viewFile/131521/121119
Arbonnier M. 2000. Arbres, Arbustes et Lianes des Zones Sèches d'Afrique de l'Ouest. CIRAD-MNHN : MontpellierParis, France; 576 p.

Beaune David, Bretagnolle François, Bollache Loïc, Bourson Chloé, Gottfried Hohmann, Barbara Fruth, 2013. «Les services écologiques des bonobos (Pan paniscus) », Revue de primatologie, 5, document 59. Consulté le 11 octobre 2018. http://journals.openedition.org/primatol ogie/1641; DOI : 10.4000/primatologie. 1641

Berg CC, Hijman MEE, Weerdenburg JC, A. 1985. Flore du Gabon 26. Moraceae. Ed. Muséum National d'Histoire Naturelle : Paris, France; 276 p.

Berg CC, Wiebes JT. 1992. African fig trees and fig wasps. Koninklijke Nederlandse Akademie van Wetenschappen: Amsterdam; 298 p.

Berhaut J. 1967. Flore du Sénégal (2e édn). Clairafrique : Dakar, Sénégal; 485 p.

Bosdeveix Robin, 2016. Entre classifications fonctionnelle et phylogénétique : le groupe des végétaux. Thèse Université Paris Diderot - Paris 7- Université Sorbonne Paris Cité. École Doctorale «Savoirs scientifiques ». Laboratoire de Didactique André Revuz (LDAR) 644 p. https://tel.archives-ouvertes.fr

Burrows J, Burrows S. 2003. Figs of southern \& south-central Africa. Umdaus Press, Hatfield. 379 p.

Clement Wendy L, Weiblen George D. 2009. Morphological Evolution in the Mulberry Family (Moraceae). Systematic Botany, 33(4): 530-552. http://geo.cbs.umn.edu/Clement\&Weibl en2009.pdf

Cottee-Jones H, Eden W. Bajpai Omesh Chaudhary, Lal, Whittaker, Robert, 2016. The Importance of Ficus (Moraceae) Trees for Tropical Forest Restoration. BIOTROPICA, 48(3): 413-419. DOI : 10.1111/btp.12304.

https://www.researchgate.net/publication/2 92677254_The_Importance_of_Ficus_Mo 
raceae_Trees_for_Tropical_Forest_Restor ation

Dagnelie P. 2011-2013. Statistique théorique et appliquée. In L'analyse de la variance à un critère de classification. 3e édit., Editions De Boeck, à Louvain-la-Neuve, Belgique. 736 p.

Dhungana Priya, Purnima Devi SK. Borthakur. 2015. Morphology and distribution of the genus Ficus Linnaeus (Moraceae) in Upper Assam, India. Pleione, $\quad$ 9(1):1http://www.academia.edu/13730216/Morp hology_and_distribution_of_the_genus_Fi cus_Linnaeus_Moraceae_in_Upper_Assa m_India

Diop D. 2013. Contribution à l'étude biosystématique des espèces du genre Ficus au Sénégal. Thèse d'Etat, Faculté des sciences et techniques, Département de Biologie végétale, UCAD. Sénégal. 178 p.

Gaaliche Badii, Saddoud Olfa, Mars Messaoud. 2012. Morphological and Pomological Diversity of Fig (Ficus carica L.) Cultivars in Northwest of Tunisia. International Scholarly Research Network (ISRN) Agronomy Volume 2012, 9p. http://dx.doi.org/10.5402/2012/326461

Gbaguidi AA, Assogbap DM, Yedomonhan H, Dansi A. 2015. Caractérisation agromorphologique des variétés de niébé cultivées au Bénin. Int. J. Biol. Chem. Sci., 9(2): 1050-1066. DOI : https://www.ajol.info/index.php/ijbcs/artic le/download/119860/109318

Hargreaves P. 2006. Vegetative morphology for species identification of tropical trees: Family distribution. Cerne, Lavras, Brésil 12, n. $1, \quad$ p.1-7. http://www.redalyc.org/pdf/744/74412101. pdf

Harrison RD, Rønsted N, Xu L, Rasplus J-Y. Cruaud A. 2012. Evolution of Fruit Traits in Ficus Subgenus Sycomorus (Moraceae): To What Extent Do Frugivores Determine Seed Dispersal Mode? PLoS ONE, 7(6): e38432.

DOI:10.1371/journal.pone.0038432

Iroka Chisom F, Clement U, Okeke, Alex I, Izund, Nkumah C, Okereke Bio Louis Nyanayo, Kenneth U Ekwealor. 2015.
Taxonomic Significance of Morphological Characters in the Species of Stachytarpheta Found in Awka, Nigeria. International Journal of Plant \& Soil Science, $\quad \mathbf{8 ( 3 ) : 1 - 6 .}$ DOI: $10.9734 / \mathrm{IJPSS} / 2015 / 19409$

Knapp Wesley M, Naczi Robert FC. 2008. Taxonomy. Morphology. and Geographic Distribution of Juncus longii (Juncaceae). Systematic Botany, 33(4): 685-694. https://doi.org/10.1600/036364408786500 145

Kumar A, Bajpai O, Mishra AK, Sahu, N, Beher SK, Chaudhary LB. 2011. Assessment of diversity in the genus Ficus L. (Moraceae) of Katerniaghat Wildlife Sanctuary, Uttar Pradesh, India. Am. J. Pl. Sci., 2(1): 78-92. DOI: 10.4236/ajps.2011.21011

Lansky EP, Paavilainen HM. 2017. Figs: The Genus Ficus. CRC Press: London; 416 p.

Lebrun Jean-Pierre, Stork Adélaïde L. 1992. Enumération des Plantes à Fleurs d'Afrique Tropicale (Vol. II) : Chrysobalanaceae à Apiaceae. Conservatoire et Jardin botaniques de la Ville de Genève, Belgique; 257 p.

Loutfy MHA, Karakish EAK 1, SF. Khalifa ERA. Mira. 2005. Numerical Taxonomic Evaluation of Leaf Architecture of Some Species of Genus Ficus L. International Journal of Agriculture \& Biology, 7(3), 352-357.

http://www.fspublishers.org/published_pa pers/62089_.pdf

Mat Nashriyah, Nurrul Akmar Rosni, Nor Zaimah Ab Rashid, Norhaslinda Haron, Zanariah Mohd Nor, Nur Fatihah Hasan Nudin, ABD Ghani Yunus, Abdul Manaf Ali, 2012. Leaf Morphological Variations and Heterophylly in Ficus deltoidea Jack. Sains Malaysiana, 41(5) 527-538. http://journalarticle.ukm.my/4464/1/02\%2 520Nashriyah\%2520Mat.pdf

Metre Vishakha, Jayshree Ghorpade, 2013. An Overview of the Research on Texture Based Plant Leaf Classification. International Journal of Computer Science and Network (IJCSN), 2(3). https://arxiv.org/pdf/1306.4345 
Ngom Ablaye, Mbaye Mame Samba, Barnaud Adeline, Aboubacry Kane, Ngasoumana Ba, Madiop Gueye, Abdoul Aziz Camara , Yacine Badiane Ndour ,Kandioura Noba, 2016. Révision du genre Digitaria Haller (Poaceae) au Sénégal : proposition d'une clé de détermination pour une meilleure identification des espèces. Int. J. Biol. Chem. Sci., 10(1): 58-86. DOI : http://dx.doi.org/10.4314/ijbcs.v10i1.6

Nur Fatihah Hasan Nudin, Mat Nashriyah, Abdul Rashid Nor Zaimah, Mahmud Khairil, Abdul Manaf Ali, 2014. Leaf morphology and anatomy of 7 varieties of Ficus deltoidea (Moraceae). Turk J. Bot., 38: 677-685. DOI: 10.3906/bot-1301-7

Nwokeocha Chinyere Constance, 2015. Botanical indices of ploidy levels in some African accessions of Oryza punctata Kotschy ex Steud. Int. J. Biol. Chem. Sci., 9(1):35-47. http://dx.doi.org/10.4314/ijbcs.v9i1.4

Oladipo O, Temitope Illoh H. Chukwuma. 2010. Pollen morphology and diversity in some Nigerian species of Jatropha L. (Euphorbiaceae). Int. J. Biol. Chem. Sci., 4(3):546-554.

DOI: https://www.ajol.info/index.php/ijbcs/artic le/viewFile/60448/48677

Oluyinka A, Akinkunmi OY, Akintoye HA, Shokalu AO. 2014. Rooting, growth and sustainability of yellow Ficus (Ficus retusa 'Nitida') as affected by growth media under nursery conditions Olusola. Int. J. Biol. Chem. Sci., 8(5):2071-2080. DOI: https://dx.doi.org/10.4314/ijbcs.v8i5.13

Polat A, Aytekin Caliskan O. 2008. Fruit characteristics of table fig (Ficus carica) cultivars in subtropical climate conditions of the Mediterranean region. New Zealand
Journal of Crop and Horticultural Science, $\quad 36(2)$ : 107-115. DOI: https://doi.org/10.1080/011406708095102 26

Puig Henri, Barthélémy Daniel, Sabatier Daniel, 2003. Clé d'identification à espèces arborées de Guyane. Rev. For. Fr., LV(numéro special): 84-100. DOI: https://agritrop.cirad.fr/523831/1/ID52383 1.pdf

R Core Team, 2018. R: A language and environment for statistical computing. $\mathrm{R}$ Foundation for Statistical Computing: Vienna, Austria. https://www.Rproject.org/.

Semde Kadidia, Madjelia Cangré Ebou Dao, Boukary Ousmane Diallo, Souleymane Ganaba, 2015. Existerait-il au sein des peuplements de Lannea microcarpa des individus à caractères botaniques différents dans la zone de Zorgho (Burkina Faso)? Int. J. Biol. Chem. Sci., 9(6): 26232632.

DOI: http://dx.doi.org/10.4314/ijbcs.v9i6.9

Shu Shu R. 2003. FICUS Linnaeus. Flora of China, 5: 37-71. DOI: http://flora.huh.harvard.edu/china/PDF/PD F05/Ficus.pdf

Sonibare Mubo, A., Adeniyi, A. Jayeola, Adeyemi Egunyomi. 2004. A morphometric analysis of the genus Ficus Linn. (moraceae). African Journal of Biotechnology, 3(4): 229-235. DOI: https://doi.org/10.5897/AJB2004.0002043

Zhekun Zhou, Michael Gilbert G. 2003. MORACEAE. Flora of China, 5: 21-73. flora.huh.harvard.edu/china/mss/volume05 /Moraceae.pdf 